\title{
CONTINUITY AND SUMMABILITY FOR DOUBLE FOURIER SERIES*
}

\author{
BY \\ J. J. GERGEN AṆD S. B. LITTAUER†
}

1.1. Introduction. The object in this paper is, first, to consider two extensions to double series of Riesz's theorem $\ddagger$ on the equivalence of the Riesz and Cesàro methods of summation for simple series, $\S$ and, secondly, to consider three extensions to double Fourier series of Hardy and Littlewood's theorem, $\|$ as refined by Paley, $\rrbracket$ Bosanquet, ${ }^{* *}$ and Wiener, $\dagger \dagger$ on the equivalence of continuity in the mean of a function and the summability of its Fourier series. We consider the question of summability in Part I and that of continuity and summability in Part II. The results in Part II are based on those in Part $\mathrm{I}$.

\section{PART I}

2.1. Extensions of Riesz's Theorem. We consider here a double series

$$
\sum_{m, n=0}^{\infty} a_{m, n} .
$$

The definitions for Cesàro and Rieszian summability of this series are analogous to those for simple series. $\ddagger$ Let $-1<\alpha,-1<\beta$. Let $m, n$ be integers, positive or 0 . Let

* Presented to the Society, October 29, 1932; received by the editors May 9, 1934, and, in revised form, January 29, 1935.

$\dagger$ The first results of this paper were obtained while Dr. Gergen was Peirce instructor at Harvard University, and Dr. Littauer was National Research Fellow, also at Harvard University.

$\ddagger$ For a statement of this theorem, its proof, and references, see Hobson, 8, pp. 90-98.

Numbers in bold face type refer to the bibliography at the end of this paper.

$\S$ One extension of Riesz's theorem has been given by Merriman, 11, p. 526. Merriman's theorem is that, if $0 \leqq \alpha, 0 \leqq \beta$, if each column, $\sum_{m=0}^{\infty} a_{m, n}$, of the series (2.11) is summable by Cesàro or by Rieszian means of order $\alpha$, and if each row, $\sum_{n=0}^{\infty} a_{m, n}$, is summable by Cesàro or by Rieszian means of $\operatorname{order} \beta$, then the series is summable $(C ; \alpha, \beta)$ to sum $s$ if, and only if, it is summable $(R ; \alpha, \beta)$ to sum $s$. This theorem, which is plainly contained in Theorem II, is not very satisfactory in treating double Fourier series. Merriman's proof like ours is based on Hobson's proof of Riesz's theorem, but it takes a different form from ours.

|| Hardy and Littlewood, 5, p. 70.

I Paley, 14, p. 180 and p. 190.

** Bosanquet, 3, p. 147 and p. 153.

†† Wiener, 18, and 19, p. 78.

¥‡ Cesàro means for double series have been considered by many authors. Among the earlier of these might be mentioned Moore, 13, and Young, 20. In addition to Merriman's paper, 11, might be mentioned Mears' paper, 10, in connection with Rieszian summability. 


$$
C_{\alpha, \beta}(m, n)=S_{\alpha, \beta}(m, n) /\left\{\left(\begin{array}{c}
\alpha+m \\
m
\end{array}\right)\left(\begin{array}{c}
\beta+n \\
n
\end{array}\right)\right\},
$$

where

$$
\begin{aligned}
S_{\alpha, \beta}(m, n) & =\sum_{p=0}^{m}\left(\begin{array}{c}
\alpha+m-p \\
m-p
\end{array}\right) \sum_{q=0}^{n}\left(\begin{array}{c}
\beta+n-q \\
n-q
\end{array}\right) a_{p, q}, \\
\left(\begin{array}{l}
a \\
b
\end{array}\right) & =\frac{\Gamma(a+1)}{\Gamma(b+1) \Gamma(a-b+1)} .
\end{aligned}
$$

Then the series (2.11) is summable by Cesàro means of order $(\alpha, \beta)$, or, more shortly, is summable $(C ; \alpha, \beta)$ to sum $s$ if $C_{\alpha, \beta}(m, n) \rightarrow s$ as $(m, n) \rightarrow(\infty, \infty)$.

On the other hand, let

$$
R_{\alpha, \beta}(x, y)=x^{-\alpha} y^{-\beta} \sigma_{\alpha, \beta}(x, y)=\sum_{p<x}(x-p)^{\alpha} \sum_{q<y}(y-q)^{\beta} a_{p, q} .
$$

Then, the series (2.11) is summable by Rieszian means of order $(\alpha, \beta)$, or summable $(R ; \alpha, \beta)$, to sum $s$ if $R_{\alpha, \beta}(x, y) \rightarrow s$ as $(x, y) \rightarrow(\infty, \infty)$.

Now the natural extension of Riesz's theorem is that, if $0 \leqq \alpha, 0 \leqq \beta$, then the series (2.11) is summable $(R ; \alpha, \beta)$ to sum $s$ if, and only if, it is summable $(C ; \alpha, \beta)$ to sum $s$. This result is however in question. In our extensions we find it essential to introduce additional conditions. In the first we use the idea of ultimate boundedness, and in the second, that of ordinary boundedness. We say that the series is bounded [ultimately bounded] $(R ; \alpha, \beta)$ if $R_{\alpha, \beta}(x, y)$ is bounded independently of $x, y$ for $0<x, 0<y$ [sufficiently large $x, y$ ]. Similar definitions hold for Cesàro summability, the condition $0<x, 0<y$ being replaced by $0 \leqq m, 0 \leqq n$. When first presented for publication this paper contained no reference to ultimate boundedness, and accordingly, it contained neither Theorem I nor VI. The truth of Theorem I and one of the type of Theorem VI was conjectured by the referee, Professor Szász, who kindly communicated his ideas to the authors. It was his suggestion as to the possible use of Agnew's fundamental lemma, ${ }^{*}$ Lemma 4 below, that directed our efforts in the proofs of these theorems.

Our extensions of Riesz's theorem are as follows:

TheOREM I. Let $0 \leqq \alpha, 0 \leqq \beta$. Then (a) the series $(2.11)$ is summable $(C ; \alpha, \beta)$ to sum $s$ if it is ultimately bounded $(C ; \alpha, \beta)$ and if it is summable $(R ; \alpha, \beta)$ to sum $s$. Moreover, (b) the series is summable $(R ; \alpha, \beta)$ to sum $s$ if it is ultimately bounded $(R ; \alpha, \beta)$ and is summable $(C ; \alpha, \beta)$ to sum $s$.

* For this lemma see Agnew, 1, p. 649. For theorems and references to theorems of the same general type as Theorem I, see Agnew, 1 and 2. 
Theorem II. Let $0 \leqq \alpha, 0 \leqq \beta$. Then (a) the series (2.11) is bounded $(C ; \alpha, \beta)$ if, and only if, it is bounded $(R ; \alpha, \beta)$. In addition, (b) if the series is bounded either $(C ; \alpha, \beta)$ or $(R ; \alpha, \beta)$, it is summable $(C ; \alpha, \beta)$ to sum $s$ if, and only if, it is summable $(R ; \alpha, \beta)$ to sum $s$.

The second part of Theorem II is of course a corollary of the first part and Theorem I. The proofs of Theorem I and part (a) of Theorem II are based on the lemmas of $\S \S 3.2$ to 3.5. The last of these is Agnews' lemma; the other three are on simple series and are modeled, to some extent, after some given by Hobson in his proof of Riesz's theorem. Hobson's lemmas in general are not sufficiently precise for our purposes. Incidentally, we might point out two results which follow from our lemmas but do not seem to be in the literature. The first is the analogue of part (a) of Theorem II, and the second is to the effect that a series of functions $\sum_{m=0}^{\infty} a_{m}(x)$ is uniformly summable on the interval $a \leqq x \leqq b$ by Cesàro means of order $\alpha, 0 \leqq \alpha$, if, and only if, it is uniformly summable there by Rieszian means of order $\alpha$.

3.1. Lemmas for Theorems I and II. In these lemmas and throughout the rest of the paper we suppose that $x, y$ are positive numbers, that $m, n, p, q$ are integers, positive or 0 , and that $M$ denotes a number independent of those of the variables $x, y, m, n, p, q$ with which we are concerned at the moment. The range for these variables is understood to be

$$
0<x, \quad 0<y, \quad 0 \leqq m, \quad 0 \leqq n, \quad 0 \leqq p, \quad 0 \leqq q,
$$

or that part of this range indicated.

In Lemmas 1 to 3 we consider a series

$$
\sum_{m=0}^{\infty} A_{m} \text {. }
$$

Here $M$ is understood to be independent of the values of the $A$ 's. We denote by $k$ a fixed positive number, by $K$, the largest integer less than $k$, and by $\mu$, the largest integer less than $x$. We write

$$
S_{a}(m)=\sum_{p=0}^{m}\left(\begin{array}{c}
a+m-p \\
m-p
\end{array}\right) A_{p}, \quad \sigma_{a}(x)=\sum_{p<x}(x-p)^{a} A_{p} .
$$

We define $\left(\begin{array}{c}m \\ p\end{array}\right)$ as 0 for $p=m+1, m+2, \cdots$, and set

$$
\begin{array}{lc}
e(x)=\sum_{p<x}(-1)^{p}\left(\begin{array}{c}
K+1 \\
p
\end{array}\right)(x-p)^{k}, & \lambda=k-K, \\
E(x)=e(x)-\Gamma(k+1)\left(\begin{array}{c}
\mu+\lambda-1 \\
\mu
\end{array}\right), & T(x)=\sigma_{k}(x)-\Gamma(k+1) S_{k}(\mu) .
\end{array}
$$


3.2. We consider first $E(x)$. We have

LEMma 1 . If $k$ is an integer then $E(x)$ is bounded for all $x$ and vanishes for $k<x$. If $k$ is not an integer then

$$
|E(x)| \leqq M(x+1)^{\lambda-2} .
$$

For $K+1<x$ we have*

$$
\begin{aligned}
\mid e(x)- & k(k-1) \cdots(k-K) x^{\lambda-1} \mid \\
& =M \int_{x-1}^{x} d u_{1} \int_{u_{1}-1}^{u_{1}} d u_{2} \cdots \int_{u_{K^{-1}}}^{u_{K}}\left(u_{K+1}^{\lambda-1}-x^{\lambda-1}\right) d u_{K+1} \\
& \leqq M\left|(x-K-1)^{\lambda-1}-x^{\lambda-1}\right| .
\end{aligned}
$$

If $k$ is an integer this is 0 , and the lemma follows in this case. If $k$ is not an integer it is $O\left(x^{\lambda-2}\right)$ as $x \rightarrow \infty$. Accordingly, since $\dagger$

$$
\begin{aligned}
k(k-1) \cdots(k-K) \mu^{\lambda-1}-\Gamma(k+1)\left(\begin{array}{c}
\mu+\lambda-1 \\
\mu
\end{array}\right) & =O\left(x^{\lambda-2}\right), \\
x^{\lambda-1}-\mu^{\lambda-1} & =O\left(x^{\lambda-2}\right)
\end{aligned}
$$

as $x \rightarrow \infty$, the lemma follows in this case also.

3.3. We turn now to

LEMMA 2. We have

$$
\left|\sigma_{k}(x)\right| \leqq M \max _{m<x}\left|S_{k}(m)\right| .
$$

In addition, corresponding to each positive integer $m_{0}$, we can write

$$
\sigma_{k}(x)=\sum_{p<m} B_{p}(x) A_{p}+H(x)
$$

for $m_{0}<x$, where the $B$ 's are independent of the $A$ 's,

$$
\left|B_{p}(x)\right| \leqq M \text { for } p<m_{0}<x,
$$

and

$$
|H(x)| \leqq M_{0} \max _{m_{0} \leqq m<x}\left|S_{k}(m)\right|
$$

for $m_{0}<x ; M_{0}$ being independent of $m_{0}$ as well as $x$ and the $A$ 's.

We have

* Compare Hobson, 8, p. 90.

$\dagger$ For the former see Hobson, 8, p. 91.

$\ddagger$ See Hobson, 8, (4), p. 71. 
Hence

$$
A_{p}=\sum_{q=0}^{p}(-1)^{p+q}\left(\begin{array}{c}
K+1 \\
p-q
\end{array}\right) S_{K}(q) \text {. }
$$

$$
\sigma_{k}(x)=\sum_{p<x}(x-p)^{k} \sum_{q=0}^{p}(-1)^{p+q}\left(\begin{array}{c}
K+1 \\
p-q
\end{array}\right) S_{K}(q)=\sum_{q<x} e(x-q) S_{K}(q) .
$$

On the other hand, $*$

$$
S_{k}(\mu)=\sum_{q<x}\left(\begin{array}{c}
\mu-q+\lambda-1 \\
\mu-q
\end{array}\right) S_{K}(q) .
$$

Thus,

$$
T(x)=\sum_{q<x} E(x-q) S_{K}(q) .
$$

Now, $\dagger$

Hence,

$$
S_{K}(q)=\sum_{m=0}^{q}(-1)^{q+m}\left(\begin{array}{c}
\lambda \\
q-m
\end{array}\right) S_{k}(m) .
$$

$$
\begin{aligned}
T(x) & =\sum_{q<x} E(x-q) \sum_{m=0}^{q}(-1)^{q+m}\left(\begin{array}{c}
\lambda \\
q-m
\end{array}\right) S_{k}(m) \\
& =\sum_{m<x} D(x-m) S_{k}(m),
\end{aligned}
$$

where

$$
D(x)=\sum_{n<x}(-1)^{n} E(x-n)\left(\begin{array}{l}
\lambda \\
n
\end{array}\right) .
$$

Consider $D(x)$. If $k$ is not an integer, then, since $\ddagger$

$$
\left|\left(\begin{array}{l}
\lambda \\
n
\end{array}\right)\right| \sim \frac{1}{|\Gamma(-\lambda)|} \frac{1}{n^{\lambda+1}}
$$

as $n \rightarrow \infty$, we have, by Lemma 1 ,

Hence,

$$
\begin{aligned}
|D(x)| & \leqq M\left\{\sum_{n<x / 2}+\sum_{x / 2 \leqq n<x}\right\}(x+1-n)^{\lambda-2}(n+1)^{-\lambda-1} \\
& \leqq M\left\{(x+1)^{\lambda-2}+(x+1)^{-\lambda-1}\right\} .
\end{aligned}
$$

$$
\begin{aligned}
\sum_{m<x}|D(x-m)| & \leqq M \sum_{m<x}\left\{(x-m+1)^{\lambda-2}+(x-m+1)^{-\lambda-1}\right\} \\
& \leqq M .
\end{aligned}
$$

* See Hobson, 8, (5), p. 72.

† See Hobson, 8, (6), p. 72.

$\ddagger$ See Hobson, 8, pp. 71-72. 
On the other hand, if $k$ is an integer then $D$ is bounded for all $x$ and vanishes for $k+1<x$. It follows that (3.34) is valid in this case also. We choose $M_{0}$ so that, for all $x$,

$$
\Gamma(k+1)+\sum_{m<x}|D(x-m)| \leqq M_{0} .
$$

Allowing for the moment $m_{0}$ to have one of the values $0,1, \cdots$, we set, for $m_{0}<x$,

$$
H(x)=\Gamma(k+1) S_{k}(\mu)+\sum_{m=m_{0}}^{\mu} D(x-m) S_{k}(m) .
$$

Then, for $0 \leqq m_{0}<x$,

$$
|H(x)| \leqq M_{0} \max _{m, \leqq m<x}\left|S_{k}(m)\right| .
$$

Taking $m_{0}=0, H$ reduces to $\sigma_{k}$, and the first part of the lemma follows. In addition, for $0<m_{0}<x$, we have

$$
\sigma_{k}(x)-H(x)=\sum_{m<m_{0}} D(x-m) \sum_{p=0}^{m}\left(\begin{array}{c}
k+m-p \\
m-p
\end{array}\right) A_{p}=\sum_{p<m_{0}} B_{p}(x) A_{p},
$$

where

$$
B_{p}(x)=\sum_{m=p}^{m_{0}-1} D(x-m)\left(\begin{array}{c}
k+m-p \\
m-p
\end{array}\right) .
$$

Since these $B$ 's are independent of the $A$ 's and satisfy (3.31) the second part likewise follows.

3.4. We proceed to the proof of

LEMMA 3. We have

$$
\left|S_{k}(m)\right| \leqq M \max _{x \leqq m+1}\left|\sigma_{k}(x)\right|
$$

In addition, corresponding to each positive integer $m_{0}$, we can write

$$
S_{k}(m)=\sum_{p<m 0} C_{p}(m) A_{p}+I(m)
$$

for $m_{0} \leqq m$, where the C's are independent of the A's,

$$
\begin{array}{rlrl}
\left|C_{p}(m)\right| & \leqq M & \text { for } p<m_{0} \leqq m, \\
|I(m)| \leqq M_{0} \max _{m 0 \leqq x \leqq m+1}\left|\sigma_{k}(x)\right| & \text { for } m_{0} \leqq m,
\end{array}
$$

$M_{0}$ being independent of $m_{0}$ as well as $m$ and the $A$ 's. 
In this lemma we set $\sigma_{0}(x)=\sum_{p \leq x} a_{p}$. We note then that*

$$
S_{K}(q)=\sum_{r=0}^{K} M \sigma_{r}(q) \text {. }
$$

Hence, by (3.32),

$$
\begin{aligned}
S_{k}(m) & =M \sigma_{k}(m+1)+\sum_{q=0}^{m} E(m+1-q) \sum_{r=0}^{K} M \sigma_{r}(q) \\
& =M \sigma_{k}(m+1)+\sum_{r=0}^{K} M \sum_{q=0}^{m} E(m+1-q) \sigma_{r}(q) .
\end{aligned}
$$

Accordingly, it is enough to prove the lemma with $S_{k}(m)$ replaced by

$$
T_{r}(m)=\sum_{q=0}^{m} E(m+1-q) \sigma_{r}(q),
$$

where $r=0,1, \cdots$ or $K$.

We set

$$
W(x)=\int_{\mu}^{x}(x-t)^{\lambda-1} \sigma_{K}(t) d t,
$$

integrate by parts $K$ times, and take $x=q+1 /(K+1), q+2 /(K+1), \cdots$, $q+1$, successively. We get, for $p=1,2, \cdots, K+1$,

$$
\begin{aligned}
& \{(K+1) / p\}^{\lambda} W\{q+p /(K+1)\}=\sum_{n=0}^{K} \alpha_{n} p^{n} \sigma_{K-n}(q), \\
& \alpha_{n}(K+1)^{n} \lambda \cdots(\lambda+n) \cdot(K-n) !=K ! .
\end{aligned}
$$

Noting that for $1 \leqq K$ the determinant of the $\sigma$ 's in these equations is a nonzero multiple of the Vandermonde formed with the numbers $1,2, \cdots, K+1$, we see that $\sigma_{r}(q)$ can be expressed in the form

$$
\sigma_{r}(q)=\sum_{i=1}^{K+1} M W\{q+i /(K+1)\} .
$$

We thus have

$$
T_{r}(m)=\sum_{i=1}^{K+1} M \sum_{n=0}^{m} E(m+1-q) W\{q+i /(K+1)\} .
$$

Hence, it is enough to prove the lemma with $S_{k}(m)$ replaced by

$$
U(m)=\sum_{n=0}^{m} E(m+1-q) W(q+\xi),
$$

* Compare Hobson, 8, p. 91. 
where $\xi$ is a fixed number satisfying $0<\xi \leqq 1$.

We have*

$$
W(x)=M \sigma_{k}(x)+M \int_{0}^{\mu} \sigma_{k}^{\prime}(v) \psi(v, x),
$$

where $\psi$ remains positive and never increases as $v$ increases from 0 to $\mu$, and

$$
\psi(0, x) \leqq M .
$$

Allowing $m_{0}$ to have one of the values $0,1, \ldots$, and denoting by $M_{0}$ a number independent of $m, m_{0}$ and the $A$ 's, we can then write

$$
\begin{aligned}
U_{1}= & \sum_{q=m_{0}}^{m} E(m+1-q) W(q+\xi)=M_{0} \sum_{q=m_{0}}^{m} E(m+1-q) \int_{0}^{m_{0}} \sigma_{k}^{\prime}(v) \psi(v, q+\xi) d v \\
& +\sum_{q=m_{0}}^{m} E(m+1-q)\left\{M_{0} \sigma_{k}(q+\xi)+M_{0} \int_{m}^{q} \sigma_{k}^{\prime}(v) \psi(v, q+\xi) d v\right\} \\
= & U_{2}+U_{3},
\end{aligned}
$$

say. Now, applying the second mean-value theorem, we have

$$
\begin{aligned}
\left|U_{3}\right| & \leqq M_{0} \max _{m_{0}+\xi \leqq x \leqq m+1}\left|\sigma_{k}(x)\right| \cdot \sum_{q=m_{0}}^{m}|E(m+1-q)| \\
& \leqq M_{0} \max _{m_{0}+\xi \leqq x \leqq m+1}\left|\sigma_{k}(x)\right| .
\end{aligned}
$$

From this inequality the first part of the lemma follows on taking $m_{0}=0$. In addition we can obtain the second part.

We have, for $0<m_{0} \leqq m$,

$$
U-U_{3}=\sum_{q<m_{0}} E(m+1-q) W(q+\xi)+U_{2}=U_{4}+U_{2},
$$

say. Now

$$
\begin{aligned}
U_{4} & =\sum_{q<m_{0}} E(m+1-q) \int_{q}^{q+\xi}(q+\xi-t)^{\lambda-1} \sigma_{K}(t) d t \\
& =\sum_{p<m_{0}} A_{p} \sum_{p \leqq q<m_{0}} E(m+1-q) \int_{q}^{q+\xi}(q+\xi-t)^{\lambda-1}(t-p)^{K} d t,
\end{aligned}
$$

and it is plain that the coefficient of $A_{p}$ here satisfies (3.41). On the other hand,

$$
U_{2}=M \sum_{q=m_{0}}^{m} E(m+1-q) \int_{0}^{m_{0}} \psi(u, q+\xi) \sum_{p<u}(u-p)^{k-1} A_{p} d u
$$

* See Hobson, 8, pp. 94-95. 


$$
=M \sum_{p<m_{0}} A_{p} \sum_{q=m_{0}}^{m} E(m+1-q) \int_{p}^{m_{0}}(u-p)^{k-1} \psi(u, q+\xi) d u,
$$

and we have, for $p=0,1, \cdots, m_{0}-1$,

$$
\begin{gathered}
\left|\sum_{q=m_{0}}^{m} E(m+1-q) \int_{p}^{m_{0}}(u-p)^{k-1} \psi(u, q+\xi) d u\right| \\
\leqq M \sum_{q=m_{0}}^{m}\left|E(m+1-q) \psi\left(m_{0}, q+\xi\right)\right| \leqq M .
\end{gathered}
$$

The lemma follows.

3.5. We consider finally

LEMMA 4. Let $m_{0}$ be a positive integer. Let $g_{p}(x), G_{p}(y), p=0,1, \cdots, m_{0}-1$, be defined for sufficiently large values of their arguments. For each $p$ let

$$
g_{p}(x)=o(1)
$$

as $x \rightarrow \infty$; and for sufficiently large $x, y$ let

$$
\left|\sum_{p<m_{0}} g_{p}(x) G_{p}(y)\right| \leqq F(x),
$$

where $F$ is independent of $y$. Then

$$
\sum_{p<m_{0}} g_{p}(x) G_{p}(y)=o(1) \quad \text { as } \quad(x, y) \rightarrow(\infty, \infty) .
$$

This lemma with $x, y$ replaced by integral variables is the lemma of Agnew previously cited. It is plain that the lemma is likewise valid when $x$ or $y$ is replaced by an integral variable. We shall have occasion to use it in various forms.

The lemma in the general case is an immediate corollary of Agnew's result. For suppose that (3.51) is false. Then there exist a positive $\epsilon$ and two sequences of numbers $\left\{x_{m}\right\},\left\{y_{n}\right\}, m, n=1,2, \cdots$, such that $x_{m} \rightarrow \infty$ as $m \rightarrow \infty, y_{n} \rightarrow \infty$ as $n \rightarrow \infty$, and

$$
\left|\sum_{p<m_{0}} g_{p}\left(x_{m}\right) G_{p}\left(y_{m}\right)\right| \geqq \epsilon .
$$

But, by Agnew's result,

$$
\sum_{p<m_{0}} g_{p}\left(x_{m}\right) G_{p}\left(y_{n}\right)=o(1)
$$

as $(m, n) \rightarrow(\infty, \infty)$. This gives us the contradiction.

4.1. Proof of Theorem I. As the proofs of (a) and (b) are similar in character we shall confine ourselves to the proof of (a). In addition, we shall as- 
sume that $0<\alpha, 0<\beta$. When $\alpha=\beta=0$ there is nothing to prove, and when one and not the other is 0 the proof requires similar steps to the following, but fewer of them.

To begin with we note that we can suppose that $s=0$. In fact, for either type of summability, the series (2.11) is summable to sum $s$ [ultimately bounded] if, and only if, the series

$$
\sum_{m, n=0}^{\infty} b_{m, n}, \quad b_{0,0}=a_{0,0}-s, \quad b_{m, n}=a_{m, n} \text { for } 0<m^{2}+n^{2},
$$

is summable to sum 0 [ultimately bounded] by means of the same order. The theorem for $s \neq 0$ is then a consequence of the theorem for $s=0$.

We now set

$$
P(m, y)=\sum_{p=0}^{m}\left(\begin{array}{c}
\alpha+m-p \\
m-p
\end{array}\right) \sum_{n<y}(y-n)^{\beta} a_{p, n},
$$

and proceed to show that

$$
P(m, y)=o\left(m^{\alpha} y^{\beta}\right) \text { as } \quad(m, y) \rightarrow(\infty, \infty) .
$$

Let $0<\epsilon$ be arbitrarily small. Then, denoting by $M_{0}$ the constant of (3.42), we select an $M$ and a positive integer $m_{0}$ so that

$$
\left|S_{\alpha, \beta}(m, n)\right| \leqq M m^{\alpha} n^{\beta}, \quad M_{0}\left|\sigma_{\alpha, \beta}(x, y)\right| \leqq \epsilon x^{\alpha} y^{\beta},
$$

the former for $m_{0} \leqq m, m_{0} \leqq n$, and the latter for $m_{0} \leqq x, m_{0} \leqq y$.

Next we apply Lemma 3. We can write, for $m_{0} \leqq m$,

$$
\begin{aligned}
P(m, y) & =\sum_{p<m_{0}} C_{p}(m) \sum_{n<y}(y-n)^{\beta} a_{p, n}+I(m, y) \\
& =I_{1}(m, y)+I,
\end{aligned}
$$

say, where $C_{p}(m)$ is bounded for $p<m_{0} \leqq m$, and

$$
|I| \leqq M_{0} \max _{m_{0} \leqq x \leqq m+1}\left|\sigma_{\alpha, \beta}(x, y)\right| \leqq \epsilon(m+1)^{\alpha} y^{\beta}
$$

for $m_{0} \leqq m, m_{0} \leqq y$.

Consider $I_{1}$. By Lemma 2, we have, for $m_{0}<y$,

$$
P(m, y)=\sum_{q<m_{0}} B_{q}(y) \sum_{p=0}^{m}\left(\begin{array}{c}
\alpha+m-p \\
m-p
\end{array}\right) a_{p, q}+H(m, y),
$$

where $B_{q}(y)$ is bounded for $q<m_{0}<y$, and

$$
|H| \leqq M \max _{m, \leqq n<y}\left|S_{\alpha, \beta}(m, n)\right| \leqq M m^{\alpha} y^{\beta}
$$


for $m_{0} \leqq m, m_{0}<y$. We conclude that, for $m_{0} \leqq m, m_{0}<y$,

$$
\begin{aligned}
\left|I_{1}\right| & \leqq|P|+|I| \leqq|H|+M \sum_{p=0}^{m}\left|\left(\begin{array}{c}
\alpha+m-p \\
m-p
\end{array}\right)\right| \sum_{q<m_{0}}\left|a_{p, q}\right| \\
& \leqq M(m+1)^{\alpha} y^{\beta}+M \sum_{p=0}^{m}\left|\left(\begin{array}{c}
\alpha+m-p \\
m-p
\end{array}\right)\right| \sum_{q<m_{0}}\left|a_{p, q}\right| \leqq F(m) y^{\beta},
\end{aligned}
$$

say, where $F$ depends upon $m$ but not upon $y$. On the other hand,

$$
m^{-\alpha} C_{p}(m)=o(1)
$$

as $m \rightarrow \infty$, for $p=0,1, \cdots, m_{0}-1$. Accordingly, Lemma 4 is applicable, and it follows that

$$
m^{-\alpha} y^{-\beta} I_{1}(m, y)=o(1) \text { as }(m, y) \rightarrow(\infty, \infty) .
$$

From (4.13), (4.11) now follows. By (4.12) and (4.13), we have

$$
\lim _{(m, y) \rightarrow(\infty, \infty)}\left|m^{-\alpha} y^{-\beta} P(m, y)\right| \leqq \epsilon,
$$

and since $\epsilon$ was arbitrary, this implies (4.11).

It remains to show that (4.11) with our hypotheses implies

$$
S_{\alpha, \beta}(m, n)=o\left(m^{\alpha} n^{\beta}\right) \text { as }(m, n) \rightarrow(\infty, \infty) .
$$

Letting $\epsilon$ and $M_{0}$ have the same significance as above, we select an $M$ and a positive integer $m_{0}$ so that

$$
\left|S_{\alpha, \beta}(m, n)\right| \leqq M m^{\alpha} n^{\beta}, \quad M_{0}|P(m, y)| \leqq \epsilon m^{\alpha} y^{\beta},
$$

the former for $m_{0} \leqq m, m_{0} \leqq n$ and the latter for $m_{0} \leqq m, m_{0} \leqq y$. Then we apply Lemma 3 again. We have, for $m_{0} \leqq n$,

$$
\begin{aligned}
S_{\alpha, \beta}(m, n) & =\sum_{q<m_{0}} C_{\alpha}^{\prime}(n) \sum_{p=0}^{m}\left(\begin{array}{c}
\alpha+m-p \\
m-p
\end{array}\right) a_{p, q}+I^{\prime}(m, n) \\
& =I_{1}^{\prime}(m, n)+I^{\prime},
\end{aligned}
$$

say, where $C_{q}^{\prime}(n)$ is bounded for $q<m_{0} \leqq n$, and

$$
\left|I^{\prime}\right| \leqq M_{0} \max _{m 0 \leqq y \leqq n+1}|P(m, y)| \leqq \epsilon m^{\alpha}(n+1)^{\beta}
$$

for $m_{0} \leqq m, m_{0} \leqq n$. Now,

$$
\left|I_{1}^{\prime}\right| \leqq\left|S_{\alpha, \beta}\right|+\left|I^{\prime}\right| \leqq M m^{\alpha}(n+1)^{\beta}
$$

for $m_{0} \leqq m, m_{0} \leqq n$. Hence, by Lemma 4 , since

$$
n^{-\beta} C_{q}^{\prime}(n)=o(1)
$$


as $n \rightarrow \infty$, for $q=0,1, \cdots, m_{0}-1$, we have

$$
n^{-\alpha} n^{-\beta} I_{1}^{\prime}(m, n)=o(1)
$$

as $(m, n) \rightarrow(\infty, \infty)$. We conclude that

$$
\lim _{(m, n) \rightarrow(\infty, \infty)}\left|m^{-\alpha} n^{-\beta} S_{\alpha, \beta}(m, n)\right| \leqq \epsilon
$$

and accordingly, that (4.14) holds.

4.2. Proof of Theorem II. We have only to prove (a). We first observe that

$$
\left|S_{\alpha, \beta}(m, n)\right| \leqq M \max _{x \leqq m+1}\left|\sum_{p<x}(x-p)^{\alpha} \sum_{q=0}^{n}\left(\begin{array}{c}
\beta+n-q \\
n-q
\end{array}\right) a_{p, q}\right| .
$$

This is a consequence of Lemma 2 if $0<\alpha$ and is trivial otherwise. We next observe that, similarly,

$$
\left|\sum_{p<x}(x-p)^{\alpha} \sum_{q=0}^{n}\left(\begin{array}{c}
\beta+n-q \\
n-q
\end{array}\right) a_{p, q}\right| \leqq M \max _{y \leqq n+1}\left|\sigma_{\alpha, \beta}(x, y)\right| .
$$

Hence, by (4.21), if the series (2.11) is bounded $(R ; \alpha, \beta)$, we have

$$
\left|S_{\alpha, \beta}(m, n)\right| \leqq M(m+1)^{\alpha}(n+1)^{\beta}
$$

from which it follows that it is bounded $(C ; \alpha, \beta)$.

In the same way, if the series is bounded $(C ; \alpha, \beta)$, we have

$$
\left|\sigma_{\alpha, \beta}(x, y)\right| \leqq M(x+1)^{\alpha}(y+1)^{\beta} \text {. }
$$

It follows that $R_{\alpha, \beta}(x, y)$ is bounded for $1 \leqq x, 1 \leqq y$. But we have, for $x<1$,

$$
R_{\alpha, \beta}(x, y)=R_{\alpha, \beta}(1, y) \text {. }
$$

Hence, as a similar identity holds for $y<1$, we conclude the truth of (a).

\section{PART II}

5.1. Extension of Hardy and Littlewood's Theorem. We consider here a function $f(u, \eta)$ which is integrable* over the square $(0,0 ; \pi, \pi)$ and is even and periodic with period $2 \pi$ in each variable. We restrict our attention to the behavior of $f$ and the Fourier series of $f$,

$$
f(u, v) \sim \sum_{m, n=0}^{\infty} a_{m, n} \cos m u \cos n v,
$$

at the origin. This restriction and that as to $f$ being even-even do not of course limit the generality of our results. The series with whose summability we are concerned is then the series (2.11) where now

* All our integrals are understood to be taken in the sense of Lebesgue. 


$$
\begin{aligned}
a_{m, n}=\lambda_{m} \lambda_{n} \int_{0}^{\pi} \cos m u d u \int_{0}^{\pi} \cos n v f(u, v) d v & \\
& {\left[\lambda_{0}=1 / \pi, \lambda_{1}=\lambda_{2}=\cdots=2 / \pi\right] . }
\end{aligned}
$$

To extend the Hardy and Littlewood theorem we need a definition of fractional integration and continuity in the mean for functions of two variables. These are direct generalizations of those for functions of a single variable. Let $\phi(u, v)$ be defined for almost all $(u, v)$ in the quarter-plane $T:(0<u, 0<v)$. Let $0<a, 0<b$. Then we define $\phi_{a, b}(x, y), \phi_{a, 0}, \phi_{0, b}$ as

$$
\begin{aligned}
\Gamma(a) \Gamma(b) \phi_{a, b}(x, y) & =\int_{(0,0)}^{(x, y)}(x-u)^{a-1}(y-v)^{b-1} \phi(u, v) d(u, v), \\
\Gamma(a) \phi_{a, 0}(x, y) & =\int_{0}^{x}(x-u)^{a-1} \phi(u, y) d u, \\
\Gamma(b) \phi_{0, b}(x, y) & =\int_{0}^{y}(y-v)^{b-1} \phi(x, v) d v
\end{aligned}
$$

provided the corresponding integral exists, and as $\infty$ otherwise. We define $\phi_{0,0}(x, y)$ as

$$
\phi_{0,0}(x, y)=\phi(x, y)
$$

where $\phi$ is defined, and as $\infty$ otherwise. We call $\phi_{a, b}(x, y)$, where $0 \leqq a, 0 \leqq b$, the fractional integral of order $(a, b)$ of $\phi$ at $(x, y)$. We say that $\phi$ is continuous $(C ; a, b)$ at the origin, or, more briefly, continuous $(C ; a, b)$, with limit $s$, if $\Gamma(a+1) \Gamma(b+1) x^{-a} y^{-b} \phi_{a, b}(x, y) \rightarrow s$ as $(x, y) \rightarrow(+0,+0)$. In addition we say that $\phi$ is almost continuous $(C ; a, b)$ with limit $s$ if $\Gamma(a+1) \Gamma(b+1) x^{-a} y^{-b}$ - $\phi_{a, b}(x, y)$ coincides, except possibly on a set of measure 0 , with a function $\Phi(x, y)$ which tends to $s$ when $(x, y) \rightarrow(+0,+0)$.

In regard to these integrals we prove in $\$ 6.1$ the following theorem:*

THEOREM III. Suppose that $0 \leqq a, 0 \leqq b$ and that $\phi(u, v)$ is integrable over every rectangle $(0,0 ; x, y)$. Then $(\mathrm{a}) \phi_{a, b}(u, v)$ is integrable over every such rectangle. In addition, (b) if $a \leqq \alpha, b \leqq \beta$ and if $\phi_{\alpha, \beta}(x, y)$ is finite, then $\phi_{\alpha, \beta}(x, y)$ is equal to the fractional integral of order $(\alpha-a, \beta-b)$ of $\phi_{a, b}$ at $(x, y)$.

Our principal extension of the Hardy and Littlewood theorem involves the idea of boundedness in much the same way as Theorem II. We say that $f$ is bounded [almost bounded] $(C ; a, b)$ in a domain $D$ if $x^{-a} y^{-b} f_{a, b}$ is bounded [almost bounded] in $D$. The extension is then as follows:

* For a theorem of this type on fractional integrals of functions of a single variable, see Tonelli, 17, p. 185. 
THEOREM IV. (a) Let

$$
0 \leqq a<\alpha, \quad 0 \leqq b<\beta .
$$

Then, if for some positive $\delta, f$ is almost bounded $(C ; a, b)$ in the infinite rectangles $(0,0 ; \infty, \delta),(0,0 ; \delta, \infty)$, it follows that the series $(2.11)$ is bounded $(C ; \alpha, \beta)$. If, in addition, $f$ is almost continuous $(C ; a, b)$ with limit $s$, then the series is summable $(C ; \alpha, \beta)$ to sum $s$. (b) On the other hand, suppose that

$$
0 \leqq \alpha<a-1, \quad 0 \leqq \beta<b-1 .
$$

Then, if the series (2.11) is bounded $(C ; \alpha, \beta)$, it follows that $f$ is bounded $(C ; a, b)$ in the quarter-plane $T$. If, in addition, the series is summable $(C ; \alpha, \beta)$ to sum $s$, then $f$ is continuous $(C ; a, b)$ with limit $s .{ }^{*}$

The proof of this theorem is given in $\$ \$ 7.1$ to 10.1 . We were much influenced in our procedure by the work of Bosanquet and Paley previously cited. $\dagger$ We do not however follow one or the other of these authors completely. The proof of part (b) especially seems to involve new difficulties. In using this method of proof it is natural that we obtain relations between the order of summability and continuity analogous to those of Bosanquet and Paley.

Part (a) of the theorem is not entirely satisfactory. Conditions depending explicitly on $f$ in the fundamental square $(0,0 ; \pi, \pi)$ would be more desirable. Conditions of this type are given in the following theorem. It might be noted in particular that, when $a \leqq 1, b \leqq 1$, the boundedness condition reduces simply to $f$ being almost bounded $(C ; a, b)$ on $(0,0 ; \pi, \delta)$ and $(0,0 ; \delta, \pi)$. Part (b) we add for the sake of completeness.

* This theorem contains as a particular case a result given independently by Moore, 13, p. 96, and Young, 20, p. 181, namely, that the series $(2.11)$ is summable $(C ; 1,1)$ to sum $s$ if the conditions in (a) hold for $a=b=0$.

Another summability criterion which might be mentioned is one due to Tonelli, 16, p. 490 . Tonelli shows that the series $(2.11)$ is summable $(C ; 1,1)$ if

$$
\int_{0}^{x} d u \int_{0}^{y}|f(u, v)-s| d v=o(x, y) \quad \text { as }(x, y) \rightarrow(+0,+0)
$$

and if the integrals $\int_{0}^{\pi}|f(x, v)| d v,\left|\int_{0}^{\pi} f(u, y)\right| d u$ are almost bounded on $(0, \pi)$. This result does not seem to be contained in Theorem III. It seems likely that an extension of Tonelli's theorem, similar to the extension of Lebesgue's theorem by Hardy for simple series, can be obtained by means of the formula in Lemma 9. For references to Hardy's theorem and to Lebesgue's theorem and to similar theorems, see Kogbetliantz, 9, p. 64.

The problem of extending Hardy and Littlewood's theorem to double series was first considered by Merriman, 12.

$\dagger$ While this paper was being prepared for publication a second paper on summability was published by Bosanquet. This paper, 4, contains a proof of the essential Lemma 9 below. In his first treatment of the problem Bosanquet used a somewhat different method. 
ThEOREM V. (a) Part (a) of Theorem IV holds if we replace the rectangles $(0,0 ; \infty, \delta),(0,0 ; \delta, \infty)$ by $(0,0 ; \pi, \delta),(0,0 ; \delta, \pi)$ and assume in addition that $y^{-b} f_{m, b}(\pi, y)\left[x^{-a} f_{a, m}(x, \pi)\right]$ is almost bounded on $(0, \delta)$ for each positive odd integer $m$ less than $a[b]$. (b) On the other hand, if (5.12) holds and if the series (2.11) is bounded $(C ; \alpha, \beta)$ it follows that $y^{-b} f_{m, b}(\pi, y)\left[x^{-a} f_{a, m}(x, \pi)\right]$ is bounded for all $y[x]$ for each positive odd integer $m$ less than $a[b]$.

This proof is found in $\$ \$ 11.1$ to 12.2 . In $\$ 13.1$ we obtain with the help of the previous Lemma 4 and the lemma of $\$ 9.5$ a third extension of the Hardy and Littlewood theorem. This result is of the same type as that of Theorem I. Whether a corresponding result holds when the roles of summability and continuity are interchanged we are unable to say.

THEOREM VI. Suppose that

$$
0 \leqq \alpha<a-2, \quad 0 \leqq \beta<b-2,
$$

and that the series (2.11) is summable either $(C ; \alpha, \beta)$ or $(R ; \alpha, \beta)$ to sum $s$. Suppose also that, for a positive $\delta, f$ is bounded $(C ; a, b)$ in the square $(+0,+0$; $\delta, \delta)$. Then $f$ is continuous $(C ; a, b)$ with limits.

6.1. Proof of Theorem III. Part (a) is trivial for $a=b=0$, part (b), for $a=\alpha, b=\beta$. We shall assume in (a) that $0<a, 0<b$, and in (b), that $0<a<\alpha$, $0<b<\beta$. The other cases can be handled by similar arguments.

Let $(x, y)$ be fixed. Then the function $(x-\xi)^{a}(y-\eta)^{b} \phi(\xi, \eta)$ is integrable over $0 \leqq \xi \leqq x, 0 \leqq \eta \leqq y$. Moreover,

$$
(x-\xi)^{a}=a \int_{\xi}^{x}(u-\xi)^{a-1} d u, \quad(y-\eta)^{b}=b \int_{\xi}^{y}(v-\eta)^{b-1} d v .
$$

Hence, by Fubini's theorem, ${ }^{*}$ it is plain that the integral

$$
\int_{0}^{x} d \xi \int_{0}^{y} d \eta \int_{\xi}^{x} d u \int_{\eta}^{y}(u-\xi)^{a-1}(v-\eta)^{b-1}|\phi(\xi, \eta)| d v
$$

exists. Thus, since the integrand here is measurable over the domain $V:(0 \leqq \xi \leqq u \leqq x, 0 \leqq \eta \leqq v \leqq y)$, the integral

$$
\int_{v}(u-\xi)^{a-1}(v-\eta)^{b-1} \phi(\xi, \eta) d(\xi, \eta, u, v)
$$

exists by a theorem due to Hobson. $\nmid$ Accordingly, the integral

$$
\int_{(0,0)}^{(x, y)} d(u, v) \int_{(0,0)}^{(u, v)}(u-\xi)^{a-1}(v-\eta)^{b-1} \phi(\xi, \eta) d(\xi, \eta)
$$

* See, for example, Hobson, 7, p. 630.

$\uparrow$ See Hobson, 7, p. 631. 
exists. We conclude that (a) holds.

The proof of (b) is similar. We have, assuming $\phi_{\alpha, \beta}(x, y)$ finite, $E \phi_{\alpha, \beta}(x, y)$

$$
=\int_{0}^{x} d \xi \int_{0}^{y} d \eta \int_{\xi}^{x} d u \int_{\eta}^{y}(x-u)^{\alpha-a-1}(y-v)^{\beta-b-1}(u-\xi)^{a-1}(v-\eta)^{b-1} \phi(\xi, \eta) d v
$$

where $E=\Gamma(\alpha-a) \Gamma(\beta-b) \Gamma(a) \Gamma(b)$. Now, noting that $\psi_{\alpha, \beta}(x, y)$, where $\psi=|\phi|$, is likewise finite, we see that we can integrate first with respect to $(\xi, \eta)$. We thus get

$$
\begin{aligned}
& E \phi_{\alpha, \beta}(x, y) \\
& \quad=\int_{(0,0)}^{(x, y)}(x-u)^{\alpha-a-1}(y-v)^{\beta-b-1} d(u, v) \int_{(0,0)}^{(u, v)}(u-\xi)^{a-1}(v-\eta)^{b-1} \phi d(\xi, \eta),
\end{aligned}
$$

and this completes the proof.

7.1. Lemma on Young's functions. We divide the proof of Theorem IV into several parts, considering first a lemma in connection with the function

$$
\begin{aligned}
\gamma_{\eta}(u) \equiv & \gamma_{\eta}^{(0)}(u) \equiv(1 / \eta)\left[1-u^{2} /\{(\eta+1)(\eta+2)\}\right. \\
& \left.+u^{4} /\{(\eta+1)(\eta+2)(\eta+3)(\eta+4)\}-\cdots\right]
\end{aligned}
$$

and its derivatives $\boldsymbol{\gamma}_{\eta}^{(1)}, \boldsymbol{\gamma}_{\eta}^{(2)}, \ldots$. For $0<u$, we have

$$
\gamma_{\eta}(u)=\Gamma(\eta) u^{-\eta} C_{\eta}(u) \text {, }
$$

where

$$
C_{\eta}(u)=(1 / \Gamma(\eta)) \int_{0}^{u}(u-t)^{\eta-1} C_{0}(t) d t \quad\left[C_{0}(u)=\cos u\right]
$$

is Young's function.*

LemMa 5. Let $0<\eta$ and let $m<\eta+1$. Then $\gamma_{\eta}^{(m)}(u)$ is continuous for all $u$, and

$$
\gamma_{\eta}^{(m)}(u)=O\left(u^{-\eta}+u^{-m-2}\right) \text { as } u \rightarrow \infty .
$$

The conclusions of this lemma are familiar. $\dagger$ First, it is plain that $\gamma_{\eta}{ }^{(m)}$ is continuous for all $u$. Next, Young $\ddagger$ has proved that, if $0 \leqq \xi$, then

* Young, 21.

$\dagger$ Hardy and Littlewood, 6, p. 217, state without proof that, as $u \rightarrow \infty$,

$$
\gamma_{1+\eta}(u)=A u^{-1-\eta} \sin (u-\pi \eta / 2)+B u^{-2}+O\left(u^{-2-\eta}\right)+O\left(u^{-2}\right),
$$

where $A$ and $B$ are constants, and that asymptotic formulas for the derivatives of $\gamma_{1+\eta}$ are given by the formal derivatives of this expression. Using the method employed above, Young, 21, Hobson, 8, p. 566, and Pollard, 15, p. 212, obtain (7.13) for various cases. None of these authors treat the case $\eta<m<\eta+1$ however.

$\ddagger$ Young, 21, or see Hobson, 8, p. 565. 


$$
C_{\xi}(u)=O\left(1+u^{\xi-2}\right) \text { as } u \rightarrow \infty .
$$

Using this result, (7.11), and the fact that

$$
D_{u} C_{\xi}=C_{\xi-1}
$$

for $0<u$, and $1 \leqq \xi$, we see that (7.13) holds if $m \leqq \eta$.

Suppose then that $\eta<m<\eta+1$; we have, by (7.11), (7.14) and (7.15),

$$
\gamma_{\eta}^{(m)}(u)=O\left(u^{-\eta-1}+u^{-m-2}+u^{-\eta}\left|D_{u} C_{\eta+1-m}\right|\right)
$$

as $u \rightarrow \infty$. But, replacing $t$ by $u-t$ in (7.15), we get

$$
\Gamma(\eta+1-m) C_{\eta+1-m}(u)=\cos u \int_{0}^{u} t^{\eta-m} \cos t d t+\sin u \int_{0}^{u} t^{\eta-m} \sin t d t .
$$

Hence,

$$
D_{u} C_{\eta+1-m}=O\left\{u^{\eta-m}+\left|\int_{0}^{u} t^{\eta-m} \cos t d t\right|+\left|\int_{0}^{u} t^{\eta-m} \sin t d t\right|\right\}=O(1) .
$$

We conclude that (7.13) holds in this case. This completes the proof.

7.2. Lemmas for part (a) of Theorem IV. In these lemmas and in the proof of part (a) we suppose that $a, \alpha, b, \beta$ satisfy (5.11). We denote by $h$ the integral part of $a$. Then it is readily seen, on applying Lemma 5 , that $\left|\gamma_{\alpha+1}^{(h+1)}(t u)(t-1)^{h-a}\right|$ is integrable in $t$ over $(1, \infty)$ for $0<u$. We set

$$
H(u)=u^{h+1-a} \int_{1}^{\infty} \gamma_{\alpha+1}^{(h+1)}(t u)(t-1)^{h-a} d t .
$$

Similarly, we write $k$ for the integral part of $b$, and set

$$
K(v)=v^{k+1-b} \int_{1}^{\infty} \gamma_{\beta+1}^{(k+1)}(t)(t-1)^{k-b} d t .
$$

We put

$$
\psi_{a}=2(-1)^{h+1} /\{\pi \Gamma(h+1-a)\}, \psi_{b}=2(-1)^{k+1} /\{\pi \Gamma(k+1-b)\}, \psi=\psi_{a} \psi_{b} .
$$

We consider first $H(u)$. We have

LEMMA 6. The function $H(u)$ is bounded and measurable over $0<u$. Moreover, ${ }^{*}$ as $u \rightarrow \infty$,

$$
H(u)=O\left(u^{-\alpha-1}+u^{-h-2}\right) .
$$

\footnotetext{
* Bosanquet, 4, p. 19, obtains an asymptotic formula for $B$ by means of Cauchy's theorem.
} 
Since, for each $u, H$ is the limit as $n \rightarrow \infty$ of the sequence of continuous functions

$$
\left\{u^{h+1-a} \int_{1+1 / n}^{n} \gamma_{\alpha+1}^{(h+1)}(t u)(t-1)^{h-a} d t\right\},
$$

we have immediately that $H$ is measurable over $0<u$.

Now, for $0<u$,

$$
\begin{aligned}
|H| & =\left|u^{h+1-a}\left\{\int_{1}^{1+1 / u}+\int_{1+1 / u}^{\infty}\right\} \gamma_{\alpha+1}^{(h+1)}(t u)(t-1)^{h-a} d t\right| \\
& \leqq(h+1-a)^{-1} \max _{u \leqq t}\left|\gamma_{\alpha+1}^{(h+1)}(t)\right|+2 \max _{u \leqq t}\left|\gamma_{\alpha+1}^{(h)}(t)\right| .
\end{aligned}
$$

It follows that $B$ is bounded for $0<u$, and that, as $u \rightarrow \alpha$,

$$
\begin{aligned}
H & =O\left(u^{-\alpha-1}+u^{-h-8}+u^{-\alpha-1}+u^{-h-2}\right) \\
& =O\left(u^{-\alpha-1}+u^{-h-2}\right) .
\end{aligned}
$$

This proves the lemma.

7.3. We have next

LEMMa 7. Let $\phi(u)$ be integrable over $(0, \pi)$, even, and periodic with period $2 \pi$. For $0 \leqq \eta$ let $\phi_{\eta}(u)$ be the fractional integral of order $\eta$ of $\phi$. Then, if $0<\delta$, we have

$$
Q \equiv \int_{\delta}^{\infty}\left|H(x u) \phi_{a}(u)\right| d u \leqq M\left(x^{-\alpha-1}+x^{-h-2}\right) \int_{0}^{\pi}|\phi| d u \text { for } 1 \leqq x,
$$

where $M$ is independent of $\phi$ as well as $x$. In addition,

$$
\int_{0}^{\infty}\left|H(x u) \phi_{a}(u)\right| d u \leqq N \int_{0}^{\pi}|\phi| d u,
$$

where $N$ is independent of $\phi$.

The proofs of (7.31) and (7.32) are much the same. We consider that of the former. We denote by $M$ a number independent of $u, t, x$ and $\phi$ for $0<u$, $0<t, 1 \leqq x$.

Now, if $1 \leqq a$, then

$$
\left|\phi_{a}(u)\right| \leqq M \int_{0}^{u}(u-t)^{a-1}|\phi| d t \leqq M(u+1)^{a} S \text {, where } S=\int_{0}^{\pi}|\phi| d u .
$$

Hence, if $a=0$ or if $1 \leqq a$, 


$$
Q \leqq M S\left(x^{-\alpha-1} \sum_{m=1}^{\infty} m^{a-\alpha-1}+x^{-h-2} \sum_{m=1}^{\infty} m^{a-h-2}\right) \leqq M S\left(x^{-\alpha-1}+x^{-h-2}\right)
$$

as a consequence of Lemma 6. Thus, (7.31) holds in this case.

Suppose then that $0<a<1$. We have

$$
\begin{aligned}
Q & \leqq M \int_{\delta}^{\infty}|H| d u \int_{0}^{u}(u-t)^{a-1}|\phi| d t \\
& =M \int_{0}^{\delta}|\phi| d t \int_{\delta}^{\infty}|H|(u-t)^{a-1} d u+M \int_{\delta}^{\infty}|\phi| d t \int_{t}^{\infty}|H|(u-t)^{a-1} d u .
\end{aligned}
$$

But

Hence,

$$
\begin{aligned}
\int_{t}^{\infty}|H|(u-t)^{a-1} d u & \leqq M x^{-\alpha-1} \int_{t}^{\infty} u^{-\alpha-1}(u-t)^{a-1} d u \\
& +M x^{-h-2} \int_{t}^{\infty} u^{-h-2}(u-t)^{a-1} d u \\
& \leqq M x^{-\alpha-1} t^{a-\alpha-1}+M x^{-h-2} t^{a-h-2}
\end{aligned}
$$

$$
\begin{aligned}
Q \leqq & M x^{-\alpha-1}\left\{\int_{0}^{\delta}|\phi| d t+\int_{\delta}^{\infty}|\phi| t^{a-\alpha-1} d t\right\} \\
& +M x^{-h-2}\left\{\int_{0}^{\delta}|\phi| d t+\int_{\delta}^{\infty}|\phi| t^{a-h-2} d t\right\} .
\end{aligned}
$$

The lemma follows.

7.4. We have thirdly

LEMMA 8. Let the hypotheses of Lemma 7 hold; and let the series (3.11) be the Fourier series of $\phi$ at the origin, so that

Then

$$
A_{m}=\lambda_{m} \int_{0}^{\pi} \phi(u) \cos m u d u
$$

$$
\psi_{a} x^{\alpha+a+1} \int_{0}^{\infty} H(x u) \phi_{a}(u) d u=\sigma_{\alpha}(x)
$$

This lemma is contained in one of Bosanquet's theorems, 4, p. 22. The proof is analogous to that of Lemma 11 below. In this case the starting point is the well known formula

$$
2 x^{\alpha+1} \int_{0}^{\infty} \gamma_{\alpha+1}(x u) \phi(u) d u=\pi \sigma_{\alpha}(x),
$$

and integration by parts is carried out $(h+1)$ times. 
7.5. We have finally

Lемма 9. Under the hypotheses that $f$ is even-even, periodic with period $2 \pi$ in each variable, and integrable over $(0,0 ; \pi, \pi)$, the function $\left|H(x u) K(y v) f_{a, b}(u, v)\right|$ is integrable over $T$ and

$$
\psi x^{a+1} y^{b+1} \int_{T} H(x u) K(y v) f_{a, b}(u, v) d T=R_{\alpha, \beta}(x, y) .
$$

Applying Lemmas 7 and 8, with $\phi(u)=f(u, v)$, we have, for almost all $v$ on $(0, \pi)$,

$$
\int_{0}^{\infty}\left|H f_{a, 0}\right| d u \leqq N \int_{0}^{\pi}|f| d u
$$

where $N$ is independent of $v$, and

$$
\psi_{a} x^{\alpha+a+1} \int_{0}^{\infty} H f_{a, 0} d u=\sum_{p<x}(x-p)^{\alpha} \lambda_{p} \int_{0}^{\pi} f \cos p u d u .
$$

From (7.51), the measurability of $H f_{a, 0}$ over $(0,0 ; \infty, \pi)$, and the integrability of $f$ we deduce the existence of the integral*

$$
I_{0}=\int_{0}^{\infty}|H| d u \int_{0}^{\pi}\left|f_{a, 0}\right| d v
$$

In addition, we see that the integrals

$$
I_{q}=\int_{0}^{\infty}|H| d u \int_{0}^{\pi}\left|f_{a, 0} \cos q v\right| d v \quad(q=1,2, \cdots)
$$

exist.

Now let $E$ be the set of values $u$ on $(0, \infty)$ such that $f_{a, b}$ is integrable in $v$ over every finite interval $(0,|z|)$. Then, applying Theorem III, the complement of $E$ relative to $(0, \infty)$ is of measure 0 , and, for $u$ on $E$,

$$
\phi(v)=f_{a, 0}(u,|v|)
$$

satisfies the conditions of Lemma 7 and

$$
\phi_{b}(v)=f_{a, b}(u, v)
$$

for almost all $v$ on $(0, \infty)$. Hence, for $u$ on $E$,

$$
\int_{0}^{\infty}\left|K f_{a, b}\right| d v \leqq N \int_{0}^{\pi}\left|f_{a, 0}\right| d v
$$

* See, for example, Hobson, 8, p. 346. 
where $N$ is independent of $u$, and

$$
\psi_{b} y^{\beta+b+1} \int_{0}^{\infty} K f_{a, b} d v=\sum_{q<y}(y-q)^{\beta} \lambda_{q} \int_{0}^{\pi} f_{a, 0} \cos q v d v .
$$

From the existence of $I_{0}$ we then conclude that $\left|H K f_{a, b}\right|$ is integrable over $T$, and from (7.52) and the existence of $I_{0}, I_{1}, \cdots$, that

$$
\begin{aligned}
\psi x^{a+1} y^{b+1} \int_{T} H K f_{a, b} d T & =\psi x^{a+1} y^{b+1} \int_{0}^{\infty} H d u \int_{0}^{\infty} K f_{a, b} d v \\
& =\psi_{a} x^{a+1} y^{-\beta} \sum_{q<y}(y-q)^{\beta} \lambda_{q} \int_{0}^{\pi} \cos q v d v \int_{0}^{\infty} H f_{a, 0} d u \\
& =R_{\alpha, \beta}(x, y) .
\end{aligned}
$$

8.1. Proof of part (a) of Theorem IV. We note that in the proof of the second part we can assume that $s=0$. For, on the one hand, $f-s$ satisfies the conditions imposed upon $f$ with $s$ replaced by 0 ; and, on the other hand, the series $(2.11)$ is summable $(C ; \alpha, \beta)$ to sum $s$ if the Fourier series of $f-s$ at the origin is summable $(C ; \alpha, \beta)$ to sum 0 .

Suppose then that, corresponding to some $\epsilon$, there is a positive $d$ and an $M$ such that

$$
\left|f_{a, b}(u, v)\right| \leqq \epsilon u^{a} v^{b}, \quad\left|f_{a, b}(u, v)\right| \leqq M u^{a} v^{b},
$$

the former for almost all $(u, v)$ in $(0,0 ; d, d)$, and the latter for almost all $(u, v)$ in $(0,0 ; \infty, d)$ and in $(0,0 ; d, \infty)$. Writing

$$
\begin{aligned}
\int_{T} H(x u) K(y v) f_{a, b} d T & =\left[\int_{(0,0)}^{(d, d)}+\int_{(d, 0)}^{(\infty, d)}+\int_{(0, d)}^{(d, \infty)}+\int_{(d, d)}^{(\infty, \infty)}\right] H K f_{a, b} d(u, v) \\
& =F_{1}+F_{2}+F_{3}+F_{4},
\end{aligned}
$$

say, we have

$$
\left|F_{1}\right| \leqq \epsilon x^{-a-1} y^{-b-1} \int_{0}^{\infty}|H(u)| u^{a} d u \int_{0}^{\infty}|K(v)| v^{b} d v \leqq M x^{-a-1} y^{-b-1}
$$

as a consequence of Lemma 6,

$$
\begin{aligned}
\left|F_{2}\right| & \leqq M y^{-b-1} \int_{d}^{\infty}\left\{(x u)^{-\alpha-1}+(x u)^{-h-2}\right\} u^{a} d u \int_{0}^{\infty}|K(v)| v^{b} d v \\
& \leqq M\left(x^{-\alpha-1}+x^{-h-2}\right) y^{-b-1} \\
\left|F_{3}\right| & \leqq M x^{-a-1}\left(y^{-b-1}+y^{-k-2}\right)
\end{aligned}
$$

and, by Lemma 7 , for $1 \leqq x, 1 \leqq y$, 


$$
\begin{aligned}
\left|F_{4}\right| & \leqq M\left(y^{-\beta-1}+y^{-k-2}\right) \int_{d}^{\infty}|H(x u)| d v \int_{0}^{\pi}\left|f_{a, 0}\right| d v \\
& \leqq M\left(x^{-\alpha-1}+x^{-h-2}\right)\left(y^{-\beta-1}+y^{-k-2}\right) .
\end{aligned}
$$

We conclude that

$$
\left|R_{\alpha, \beta}(x, y)\right| \leqq M\left(1+x^{a-\alpha}+x^{a-h-1}\right)\left(1+y^{b-\beta}+y^{b-k-1}\right)
$$

for $1 \leqq x, 1 \leqq y$, and that

$$
\lim _{(x, y) \rightarrow(\infty, \infty)}\left|R_{\alpha, \beta}(x, y)\right| \leqq \epsilon \int_{0}^{\infty}|H(u)| u^{a} d u \int_{0}^{\infty}|K(v)| v^{b} d v .
$$

The proof now follows. If $f$ is almost bounded $(C ; a, b)$ in $(0,0 ; \infty, \delta)$ and in $(0,0 ; \delta, \infty)$, then (8.11) holds for some $\epsilon$, and the first part of (a) follows from the former of these inequalities. If, in addition, $f$ is almost continuous $(C ; a, b)$ with limit 0 , then (8.11) holds for each arbitrarily small $\epsilon$, and the second part follows from the latter.

9.1. Lemmas for part (b) of Theorem IV and Theorem VI. In these lemmas we suppose that $\alpha, a, \beta, b$ satisfy (5.12). We denote by $h$ the integral part of $\alpha$, and by $k$ the integral part of $\beta$. We set

$$
\begin{aligned}
\psi_{\alpha} & =(-1)^{h} /\{\Gamma(\alpha+1) \Gamma(h+1-\alpha) \Gamma(a)\}, \\
\psi_{\beta} & =(-1)^{k} /\{\Gamma(\beta+1) \Gamma(k+1-\beta) \Gamma(b)\}, \quad \psi=\psi_{\alpha} \psi_{\beta}, \\
H(u & : u^{h+1-\alpha} \int_{1}^{\infty} \gamma_{a}^{(h+2)}(t u)(t-1)^{h-\alpha} d t, K(v)=v^{k+1-\beta} \int_{1}^{\infty} \gamma_{b}{ }^{(k+2)}(t)(t-1)^{k-\beta} d t .
\end{aligned}
$$

The functions $H, K$ exist for all positive values of their arguments.

9.2. In regard to $H(u)$ we have

LEMMA 10. The function $H(u)$ is bounded and measurable for $0<u$. Moreover, as $u \rightarrow \infty$,

$$
H(u)=O\left(u^{-a}+u^{-h-3}\right) .
$$

The proof here is practically the same as that of Lemma 6 and can be omitted.

9.3. We have next

LEMMA $11 .^{*}$ Suppose that $\phi(u), \phi_{\eta}(u), 0 \leqq \eta$, are the functions of Lemma 7 . Suppose that the series (3.11) is the Fourier series of $\phi$ at the origin, and that, for some $\delta$ satisfying $0 \leqq \delta<a-1, \delta<h+2$,

* The proof of this lemma is closely analogous to a proof given by Bosanquet, 3, pp. 157-161, concerning, not the summability of a series, but the summability of an integral. In treating the series in (9.33) Bosanquet uses partial summation throughout rather than partial integration and partial summation. 


$$
\sigma_{\alpha}(u)=O\left(u^{\delta}\right) \text { as } u \rightarrow \infty .
$$

Then the function $\left|H(x u) \sigma_{\alpha}(u)\right|$ is integrable over $(0, \infty)$ and

$$
\psi_{a} x^{a+\alpha+1} \int_{0}^{\infty} H(x u) \sigma_{\alpha}(u) d u=\phi_{a}(x) .
$$

It is plain that $\left|H \sigma_{\alpha}\right|$ is integrable over $(0, \infty)$. Consider then (9.32). We have*

$$
x^{a} \sum_{m=0}^{\infty} A_{m} \gamma_{a}(m x)=\Gamma(a) \phi_{a}(x)
$$

Now, for $0<t$,

$$
\int_{0}^{t} \sigma_{m}(u) d u=\sigma_{m+1}(t) /(m+1) .
$$

Hence, denoting by $z$ a positive integer, using Abel's formula, and integrating by parts $(h+1)$ times, we have

$$
\begin{aligned}
\sum_{m=0}^{z} A_{m} \gamma_{a}(m x)= & \left\{\gamma_{a}(x z) \sigma_{0}(z+1)+\sum_{m=1}^{h+1}(-x)^{m} \gamma_{a}^{(m)}(x z) \sigma_{m}(z) / \Gamma(m+1)\right\} \\
& +\left[(-x)^{h+2} / \Gamma(h+2)\right] \int_{0}^{z} \gamma_{a}^{(h+2)}(x t) \sigma_{h+1}(t) d t \\
= & I_{1}+I_{2},
\end{aligned}
$$

say. But, since $\left|A_{m}\right| \leqq M$, we have, as $z \rightarrow \infty$,

$$
\sigma_{m}(z)=O\left\{\sum_{n<z}(z-n)^{m}\right\}=O\left(z^{m+1}\right) .
$$

Moreover, since

$$
\text { (9.34) } \sigma_{h+1}(t)=(h+1)\left(\begin{array}{l}
h \\
\alpha
\end{array}\right) \int_{0}^{t}(t-u)^{h-\alpha} \sigma_{\alpha}(u) d u \text { for } 0<t \text {, }
$$

we have, by (9.31),

$$
\sigma_{h+1}(z)=O\left\{\int_{0}^{z}(z-u)^{h-\alpha} u^{\delta} d u\right\}=O\left(z^{h+\delta+1-\alpha}\right) .
$$

Thus,

$$
I_{1}=O\left\{\sum_{m=0}^{h}\left(z^{-a}+z^{-m-2}\right) z^{m+1}+\left(z^{-a}+z^{-h-3}\right) z^{h+\delta+1-\alpha}\right\}=o(1) .
$$

* See, for example, Paley, 14, p. 190. 
It is sufficient then to show that

$$
I_{2}=\Gamma(a) \psi_{\alpha} x^{\alpha+1} \int_{0}^{z} H(x u) \sigma_{\alpha} d u+o(1) \text { as } z \rightarrow \infty
$$

Making the substitution (9.34) in $I_{2}$,

$$
\begin{aligned}
I_{2} & =\Gamma(a) \psi^{\alpha} x^{h+2} \int_{0}^{z} \gamma_{a}^{(h+2)}(x t) d t \int_{0}^{t}(t-u)^{h-\alpha} \sigma_{\alpha}(u) d u \\
& =\Gamma(a) \psi_{\alpha} x^{h+2} \int_{0}^{z} \sigma_{\alpha}(u) u^{h+1-\alpha} d u \int_{1}^{z / u} \gamma_{a}^{(h+2)}(x u t)(t-1)^{h-\alpha} d t \\
& =\Gamma(a) \psi_{\alpha} x^{\alpha+1}\left\{\int_{0}^{z} H(x u) \sigma_{\alpha}(u) d u-\int_{0}^{z} H_{1}(x, u, z) \sigma_{\alpha}(u) d u\right\},
\end{aligned}
$$

say, where

$$
H_{1}=(x u)^{h+1-\alpha} \int_{z / u}^{\infty} \gamma_{a}^{(h+2)}(x u t)(t-1)^{h-\alpha} d t .
$$

Now, for $0<u<z$,

Hence,

$$
\left|H_{1}\right| \leqq 2 x^{h-\alpha}(z-u)^{h-\alpha} \max _{x z \leqq t}\left|\gamma_{a}^{(h+1)}(t)\right| .
$$

$$
\int_{0}^{z} H_{1} \sigma_{\alpha} d u=O\left\{\left(z^{-a}+z^{-h-3}\right) \int_{0}^{z}(z-u)^{h-\alpha} u^{8} d u\right\}=o(1) .
$$

We conclude that (9.35) and, accordingly, that (9.32) holds.

9.4. We have thirdly

LEMma 12. Let the series (2.11) be bounded $(C ; \alpha, \beta)$. Then, for any $n$,

as $u \rightarrow \infty$.

$$
\zeta(u, n) \equiv \sum_{m<u}(u-m)^{\alpha} a_{m, n}=O\left(u^{\alpha}\right)
$$

We note first that, as $u \rightarrow \infty$,

$$
\zeta=O\left\{\max _{m \leqq u}\left|\sum_{p=0}^{m}\left(\begin{array}{c}
\alpha+m-p \\
m-p
\end{array}\right) a_{p, n}\right|\right\} .
$$

If $0<\alpha$, this follows from Lemma 2. If $\alpha=0$, it is trivial. Now,

$$
\sum_{p=0}^{m}\left(\begin{array}{c}
\alpha+m-p \\
m-p
\end{array}\right) a_{p, n}=\sum_{q=0}^{n}(-1)^{q}\left(\begin{array}{c}
\beta+1 \\
q
\end{array}\right) S_{\alpha, \beta}(m, n-q),
$$

* See Hobson, 8, p. 71, (4). 
where we have set

$$
\left(\begin{array}{c}
\beta+1 \\
q
\end{array}\right)=0 \text { if } \beta+1-q=-1,-2, \cdots .
$$

Thus, making use of our hypothesis,

$$
\left|\sum_{p=0}^{m}\left(\begin{array}{c}
\alpha+m-p \\
m-p
\end{array}\right) a_{p, n}\right| \leqq M(m+1)^{\alpha}(n+1)^{\beta} \sum_{q=0}^{n}\left|\left(\begin{array}{c}
\beta+1 \\
q
\end{array}\right)\right|=O\left(m^{\alpha}\right)
$$

as $m \rightarrow \infty$. The lemma follows.

9.5. We have finally

LEMMA 13. If either (a) the series (2.11) is bounded $(C ; \alpha, \beta)$, or (b) $\alpha+2<a$, $\beta+2<b$, then $\left|H(x u) K(y v) \sigma_{\alpha, \beta}\right|$ is integrable over $T$, and

$$
\psi x^{a+\alpha+1} y^{b+\beta+1} \int_{T} H(x u) K(y v) \sigma_{\alpha, \beta}(u, v) d T=f_{a, b}(x, y) .
$$

We note first that $\left|H K \sigma_{\alpha, \beta}\right|$ is integrable over $T$ in either case. If (a) holds this follows from Theorem II and Lemma 10; and if (b) holds it follows on observing that, in general,

$$
\left|\sigma_{\alpha, \beta}(x, y)\right| \leqq M x^{\alpha+1} y^{\beta+1} .
$$

Consider then (9.51). In either case, since $1<a$, the function

$$
\Phi(v) \equiv f_{a, 0}(x,|v|)
$$

is integrable in $v$ over $(0, \pi)$. Moreover, $\Phi$ is even and periodic with period $2 \pi$. Its Fourier series at the origin is

$$
\sum_{n=0}^{\infty} F_{a}^{(n)}(x), \quad \text { where } F^{(n)}(u)=\lambda_{n} \int_{0}^{\pi} \cos n v f(u, v) d v .
$$

Now, for a fixed $n, F^{(n)}$ is integrable over $(0, \pi)$, even, and periodic with period $2 \pi$. Its Fourier series at the origin is

But, if (a) holds, then

$$
\sum_{m=0}^{\infty} a_{m, n}
$$

$$
\zeta(u, n)=\sum_{m<u}(u-m)^{\alpha} a_{m, n}=O\left(u^{\alpha}\right)
$$

as $u \rightarrow \infty$. Hence in this case, by Lemma 11 , with $\phi=F^{(n)}$ and $\delta=\alpha$,

$$
F_{a}^{(n)}(x)=\psi_{\alpha} x^{a+\alpha+1} \int_{0}^{\infty} H(x u) \zeta(u, n) d n .
$$


On the other hand, in general,

$$
\zeta(u, n)=O\left(u^{\alpha+1}\right)
$$

so that, if (b) holds, we have on applying Lemma 11 with $\delta=\alpha+1$ the result (9.52) again. Accordingly, in either case the Fourier series of $\Phi(v)$ at the origin is

$$
\sum_{n=0}^{\infty} \psi_{\alpha} x^{a+\alpha+1} \int_{0}^{\infty} H(x u) \zeta(u, n) d u
$$

The lemma now follows. We have

$$
\sum_{n<v}(v-n)^{\beta} \int_{0}^{\infty} H \zeta d u=\int_{0}^{\infty} H \sigma_{\alpha, \beta}(u, v) d u .
$$

If (a) holds this is

$$
=O\left(v^{\beta} \int_{0}^{\infty}|H(x u)| u^{\alpha} d u\right)=O\left(v^{\beta}\right)
$$

as $v \rightarrow \infty$, and if (b) holds it is

$$
=O\left(v^{\beta+1} \int_{0}^{\infty}|H| u^{\alpha+1} d u\right)=O\left(v^{\beta+1}\right) .
$$

Accordingly, noting that since $1<a, 1<b, f_{a, b}(x, y)$ is finite, we have, by Theorem III and Lemma 11,

$$
\begin{aligned}
f_{a, b}(x, y)=\Phi_{b}(y) & =\psi x^{a+\alpha+1} y^{b+\beta+1} \int_{0}^{\infty} K(y v)\left\{\sum_{n<v}(v-n)^{\beta} \int_{0}^{\infty} H \zeta d u\right\} d v \\
& =\psi x^{a+\alpha+1} y^{b+\beta+1} \int_{T} H K \sigma_{\alpha, \beta} d T .
\end{aligned}
$$

10.1. Proof of part (b) of Theorem IV. Let us assume first that the series $(2.11)$ is bounded $(C ; \alpha, \beta)$. Then we have, by Lemma 13 ,

$$
\begin{aligned}
f_{a, b}(x, y) & =\psi x^{a+\alpha+1} y^{b+\beta+1} \int_{T} H(x u) K(y v) \sigma_{\alpha, b} d T \\
& =\psi x^{a} y^{b} \int_{T} H(u) K(v) \sigma_{\alpha, b}(u / x, v / y) d T
\end{aligned}
$$

and, for all $(u, v)$ in $T$,

$$
\left|H(u) K(v) x^{\alpha} y^{\beta} \sigma_{\alpha, \beta}(u / x, v / y)\right| \leqq M u^{\alpha}|H(u)| v^{\beta}|K(v)|,
$$

where $M$ is independent of $(u, v)$ as well as $(x, y)$. As the function on the right here is integrable over $T$, we conclude that $f$ is bounded $(C ; a, b)$ in $T$. 
Now let us assume in addition that the series is summable $(C ; \alpha, \beta)$ to sum $s$. Then we have

$$
\lim _{(x, y) \rightarrow(+0,+0)}\left\{H(u) K(v) x^{\alpha} y^{b} \sigma_{\alpha, \beta}(u / x, v / y)\right\}=u^{\alpha} H(u) v^{\beta} K(v)
$$

for every $(u, v)$ in $T$. We conclude from Lebesgue's theorem that

$$
\lim _{(x, y) \rightarrow(+0,+0)}\left\{f_{a, b}(x, y) /\left(x^{a} y^{b}\right)\right\}=\psi s \int_{T} u^{\alpha} H(u) v^{\beta} K(v) d T .
$$

Applying Lemma 13 to the function $f \equiv 1$, we get

Thus,

$$
\psi \Gamma(a+1) \Gamma(b+1) \int_{T} u^{\alpha} H(u) v^{\beta} K(v) d T=1 .
$$

$$
\lim _{(x, y) \rightarrow(+0,+0)}\left\{f_{a, b}(x, y) /\left(x^{a} y^{b}\right)\right\}=s /\{\Gamma(a+1) \Gamma(b+1)\} .
$$

This completes the proof.

11.1. Proof of part (a) of Theorem V. We first prove

LEMma 14. Let $\phi(u), \phi_{\eta}(u), 0 \leqq \eta$, be the functions of Lemma 7. Suppose that $0 \leqq a<\alpha$ and that, for some fixed number $K,\left|\phi_{a}(x)\right| \leqq K x^{a}$ for almost all $x$ on $(0, \pi)$,

$$
\left|\phi_{m}(\pi)\right| \leqq K \text { for each positive odd integer } m<a .
$$

Then there exists a number $N$, independent of $x, \phi, K$, such that

$$
\left|\dot{\phi}_{\alpha}(x)\right| \leqq N K x^{\alpha} \text { if } \phi_{\alpha}(x) \text { is finite. }
$$

We see that, if $a$ is a positive odd integer, then, by the continuity of $\phi_{a}$ at $x=\pi, \phi_{a}$ satisfies (11.11) with $K$ replaced by $K \pi^{a}$. We see also that, if $a<a^{\prime}$, then

$$
\begin{aligned}
\left|\phi_{a^{\prime}}(x)\right| & \leqq\left(1 / \Gamma\left(a^{\prime}-a\right)\right) \int_{0}^{x}(x-u)^{a^{\prime}-a-1}\left|\phi_{a}(u)\right| d u \\
& \leqq K x^{a^{\prime}} \Gamma(a+1) / \Gamma\left(a^{\prime}+1\right)
\end{aligned}
$$

for almost all $x$ on $(0, \pi)$. On the other hand, if $\alpha<\alpha^{\prime}$ and the conclusion holds, then

$$
\left|\phi_{\alpha^{\prime}}(x)\right| \leqq N K x^{\alpha^{\prime}} \Gamma(\alpha+1) / \Gamma\left(\alpha^{\prime}+1\right)
$$

wherever $\phi_{\alpha^{\prime}}$ is finite. Accordingly, it is enough to prove the lemma with $h<a<\alpha<h+1$, where $h$ is the integral part of $a$.

We denote by $N$ a number independent of $x, y, \phi, K$. Then

for $x \leqq \pi$, and

$$
\left|\phi_{h+1}(x)\right| \leqq N K
$$




$$
\begin{aligned}
\left|\phi_{h+1}(y)-\phi_{h+1}(x)\right| \leqq & N \int_{0}^{x}\left|(y-u)^{h-a}-(x-u)^{h-a}\right|\left|\phi_{a}\right| d u \\
& +N \int_{x}^{y}(y-u)^{h-a}\left|\phi_{a}\right| d u \\
\leqq & N K \int_{x}^{y} d t \int_{-\infty}^{x}(t-u)^{h-a-1} d u+N K \int_{x}^{y}(y-u)^{h-a} d u \\
\leqq & N K(y-x)^{h+1-a}
\end{aligned}
$$

for $x<y \leqq \pi$.

From these inequalities we conclude that

$$
\begin{aligned}
&\left|\int_{0}^{\pi}(x-u)^{\alpha-h-1} \phi_{h} d u\right| \leqq N K x^{\alpha-1} \text { for } \pi<x, \\
& \limsup _{y \rightarrow x+0}\left|\int_{y}^{\pi}(u-x)^{\alpha-h-1} \phi_{h} d u\right| \leqq N K \quad \text { for } 0 \leqq x<\pi, \\
&\left|\int_{0}^{\pi}(x+u)^{\alpha-h-1} \phi_{h} d u\right| \leqq N K(x+\pi)^{\alpha-1} .
\end{aligned}
$$

We have, for $\pi<x$,

$$
\begin{aligned}
& \left|\int_{0}^{\pi}(x-u)^{\alpha-h-1} \phi_{h} d u\right| \leqq x^{\alpha-h-1}\left|\phi_{h+1}(\pi)\right| \\
& +N \int_{0}^{\pi}(x-u)^{\alpha-h-2}\left|\phi_{h+1}(\pi)-\phi_{h+1}(u)\right| d u \\
& \leqq N K x^{\alpha-1}+N K \int_{0}^{\pi}(x-u)^{\alpha-h-2}(\pi-u)^{h+1-a} d u \leqq N K x^{\alpha-1}
\end{aligned}
$$

as can be seen by considering separately the cases $\pi<x<2 \pi, 2 \pi \leqq x$. This is (11.13).

Next, for $0 \leqq x<y<\pi$, we have

$$
\begin{aligned}
\left|\int_{y}^{\pi}(u-x)^{\alpha-h-1} \phi_{h} d u\right| \leqq & (\pi-x)^{\alpha-h-1}\left|\phi_{h+1}(\pi)-\phi_{h+1}(y)\right| \\
& +N \int_{y}^{\pi}(u-x)^{\alpha-h-2}\left|\phi_{h+1}(u)-\phi_{h+1}(y)\right| d u \\
\leqq & N K+N K \int_{y}^{\pi}(u-y)^{\alpha-a-1} d u \leqq N K .
\end{aligned}
$$

Hence, (11.14) holds.

Finally, 


$$
\begin{aligned}
\left|\int_{0}^{\pi}(x+u)^{\alpha-h-1} \phi_{h} d u\right| & \leqq(x+\pi)^{\alpha-h-1}\left|\phi_{h+1}(\pi)\right|+N \int_{0}^{\pi}(x+u)^{\alpha-h-2}\left|\phi_{h+1}\right| d u \\
& \leqq K(x+\pi)^{\alpha-1}+N K \int_{0}^{\pi}(x+u)^{\alpha-h-2} u^{h+1} d u \\
& \leqq N K(x+\pi)^{\alpha-1}
\end{aligned}
$$

as may be seen by considering the cases $x<\pi, \pi \leqq x$. This is (11.15).

Consider now (11.12). It is plain that our conclusion holds if we restrict ourselves to values of $x \leqq \pi$. We show now that it holds for $\pi<x \leqq 2 \pi$. If $\phi_{\alpha}(x)$ is finite then the integral

exists, and

$$
\Psi(x)=\int_{2 \pi-x}^{\pi}[u-(2 \pi-x)]^{\alpha-1} \phi d u
$$

$$
\Gamma(\alpha) \phi_{\alpha}(x)=\int_{0}^{\pi}(x-u)^{\alpha-1} \phi d u+\Psi(x) .
$$

If $h=0$ our conclusion then follows from (11.13) and (11.14). If $1 \leqq h$ we integrate by parts $h$ times. We get

$$
\begin{aligned}
\phi_{\alpha}(x)= & \sum_{p<h / 2} M(x-\pi)^{\alpha-2 p-1} \phi_{2 p+1}(\pi)+N \int_{0}^{\pi}(x-u)^{\alpha-h-1} \phi_{h} d u \\
& +N \int_{2 \pi-x}^{\pi}[u-(2 \pi-x)]^{\alpha-h-1} \phi_{h} d u .
\end{aligned}
$$

Applying (11.11), (11.13) and (11.14) we get the desired conclusion.

Suppose now that $2 \pi<x$. We write $x=2 n \pi+\xi$, where $n$ is a positive integer and $0<\xi \leqq 2 \pi$. Then, if $\phi_{\alpha}(x)$ is finite, so also is $\phi_{\alpha}(\xi)$, and

$$
\begin{aligned}
\Gamma(\alpha) \phi_{\alpha}(x)= & \sum_{q=0}^{n-1} \int_{0}^{\pi}\left\{(x-2 q \pi-u)^{\alpha-1}+[x-2(q+1) \pi+u]^{\alpha-1}\right\} \phi d u \\
& +\Gamma(\alpha) \phi_{\alpha}(\xi) .
\end{aligned}
$$

If $h=0$, we have, by (11.13) and (11.15),

$$
\begin{gathered}
\sum_{q=0}^{n-1}\left|\int_{0}^{\pi}\left\{(x-2 q \pi-u)^{\alpha-1}+[x-2(q+1) \pi+u]^{\alpha-1}\right\} \phi d u\right| \\
\leqq N K \sum_{q=0}^{n-1}[x-(2 q+1) \pi]^{\alpha-1} \leqq N K x^{\alpha}
\end{gathered}
$$

so that the lemma follows in this case. If $1 \leqq h$ we integrate by parts. We get, for $2 \pi<x$, 


$$
\begin{aligned}
\left|\int_{0}^{\pi}\left\{(x-u)^{\alpha-1}+(x-2 \pi+u)^{\alpha-1}\right\} \phi d u\right| & \leqq N \sum_{p<h / 2}(x-\pi)^{\alpha-2 p-1}\left|\phi_{2 p+1}(\pi)\right| \\
+N\left|\int_{0}^{\pi}(x-u)^{\alpha-h-1} \phi_{h} d u\right| & +N\left|\int_{0}^{\pi}(x-2 \pi+u)^{\alpha-h-1} \phi_{h} d u\right| \\
& \leqq N K x^{\alpha-1} .
\end{aligned}
$$

Hence,

$$
\left|\phi_{\alpha}(x)\right| \leqq N K \sum_{q=0}^{n-1}(x-2 q \pi)^{\alpha-1}+N K \leqq N K x^{\alpha},
$$

and this proves the lemma.

11.2. Turning now to the proof of (a), we select $\alpha_{0}, \beta_{0}$ so that $a<\alpha_{0}<\alpha$, $b<\beta_{0}<\beta$, and note that, as a consequence of Theorem III, $f$ is almost continuous $\left(C ; \alpha_{0}, \beta_{0}\right)$ with limit $s$. Hence, by Theorem IV, it is enough to prove that $f$ is almost bounded $\left(C ; \alpha_{0}, \beta_{0}\right)$ on $(0,0 ; \infty, \delta)$ and $(0,0 ; \delta, \infty)$.

Let $D$ denote the rectangle $(0,0 ; \infty, \delta)$. We shall show that $f$ is almost bounded $\left(C ; \alpha_{0}, \beta_{0}\right)$ on $D$. We can assume that $f$ is almost bounded $\left(C ; a, \beta_{0}\right)$ on $(0,0 ; \pi, \delta)$, and, since

$$
\int_{0}^{y}(y-v)^{\beta_{0}-1} d v \int_{0}^{\pi}(\pi-u)^{m-1}|f| d u \quad(m=1,2, \cdots)
$$

is finite for almost all $y$ on $d:(y \leqq \delta)$, that $y^{-\beta_{0}} f_{m, \beta_{0}}(\pi, y)$ is almost bounded on $d$ for each positive odd integer $m<a$.

Now there is a number $M_{0}$ and a set $e$, of measure $\delta$, of values $y$ on $d$, such that $f_{0, \beta_{0}}(u, y)$ is integrable over $(0, \pi)$, and

$$
\left|f_{a, \beta_{0}}(x, y)\right| \leqq M_{0} x^{a} y^{\beta_{0}}, \quad\left|f_{m, \beta_{0}}(\pi, y)\right| \leqq M_{0} y^{\beta_{0}}
$$

the first for almost all $x$ on $(0, \pi)$, the second for each positive odd integer $m<a$. Let $E$ denote the set of points $(x, y)$ such that $y$ belongs to $e$ and $f_{\alpha_{0}, \beta_{0}}(x, y)$ is finite. Then the complement of $E$ relative to $D$ is of measure 0 . To prove the theorem we show that $f$ is bounded $\left(C ; \alpha_{0}, \beta_{0}\right)$ on $E$.

Consider any fixed $y$ on $d$. The function

$$
\phi(u)=f_{0, \beta_{0}}(|u|, y)
$$

is integrable over $(0, \pi)$, even, and periodic with period $2 \pi$. In addition $\phi$ fulfils the remaining conditions of the lemma with $K=M_{0} y^{\beta_{0}}$. In fact, for almost all $x$ on $(0, \pi), f_{a, \beta_{0}}(x, y)$ is finite. At these points $f_{a, \beta_{0}}$ is equal to the fractional integral of $\operatorname{order}(a, 0)$ of $f_{0, \beta_{0}}$. Thus,

$$
\left|\phi_{a}(x)\right|=\left|f_{a, \beta_{0}}(x, y)\right| \leqq M_{0} x^{a} y^{\beta_{0}}
$$


for almost all $x$ on $(0, \pi)$. Moreover, $f_{m, \beta_{0}}(\pi, y)$ being finite,

$$
\left|\phi_{m}(\pi)\right|=\left|f_{m, \beta_{0}}(\pi, y)\right| \leqq M_{0} y^{\beta_{0}}
$$

for each positive odd integer $m<a$. The lemma can then be applied. We get, if $\phi_{\alpha_{0}}(x)$ is finite,

$$
\left|\phi_{\alpha_{0}}(x)\right| \leqq N M_{0} x^{\alpha_{0}} y^{\beta_{0}},
$$

where $N$ is independent of $x$ and $y$. In particular then, if $(x, y)$ is a point of $E$, we have

$$
\left|f_{\alpha_{0}, \beta_{0}}(x, y)\right|=\left|\phi_{\alpha_{0}}(x)\right| \leqq N M_{0} x^{\alpha_{0}} y^{\beta_{0}} .
$$

This completes the proof.

12.1. Proof of part (b) of Theorem V. This proof depends on

LEMMA 15. Let $\phi(u), \phi_{\eta}(u), 0 \leqq \eta$, be the functions of Lemma 7. Suppose that $1<a$, and that, for a fixed $K$,

$$
\left|\phi_{a}(x)\right| \leqq K x^{a}
$$

for $x<2 \pi$. Then, for each positive odd integer $m<a$,

$$
\left|\phi_{m}(\pi)\right| \leqq N K
$$

where $N$ is independent of $K$ and $\phi$.

We can suppose that $a=2 h+1$, where $h$ is a positive integer. Then, for $\pi<x<2 \pi$, we have

$$
\phi_{a}(x)=2 \sum_{p=0}^{h-1} \alpha_{2 p+1}(x-\pi)^{2(h-p)} \phi_{2 p+1}(\pi)+2 \alpha_{a} \phi_{a}(\pi)-\alpha_{a} \phi_{a}(2 \pi-x),
$$

where

$$
\alpha_{2 p+1}=(2 h) ! /[2(h-p)] ! .
$$

Taking $x=\pi+x_{0}^{1 / 2}, \pi+x_{1}^{1 / 2}, \cdots, \pi+x_{h-1}^{1 / 2}$ successively, where $\pi^{2}<x_{0}<x_{1}$ $<\cdots<x_{h-1}<4 \pi^{2}$, we arrive at the set of equations in the $\phi$ 's

$$
\sum_{p=0}^{h-1} \alpha_{2 p+1} x_{n}^{h-p} \phi_{2 p+1}(\pi)=\tau_{n} \quad(n=0,1, \cdots, h-1),
$$

where

$$
\left|\tau_{n}\right| \leqq 2 \alpha_{a} K(2 \pi)^{a} \text {. }
$$

As the $\alpha$ 's are independent of $\phi$ and $K$, and as the determinant of the $\phi$ 's is a non-zero multiple of the Vandermonde formed with the numbers $x_{0}, x_{1}, \cdots$, $x_{h-1}$, we see that our conclusion holds. 
12.2. Consider now the proof of (b). As a consequence of Theorem IV there is an $M_{0}$ such that, for $x<2 \pi$ and all $y$,

$$
\left|f_{a, b}(x, y)\right| \leqq M_{0} x^{a} y^{b} .
$$

For a fixed $y$, let

$$
\phi(u)=f_{0, b}(|u|, y) .
$$

Then $\phi$ satisfies the conditions of the lemma with $K=M_{0} y^{b}$. Hence,

$$
\left|f_{m, b}(\pi, y)\right|=\left|\phi_{m}(u)\right| \leqq M y^{\beta}
$$

for each positive odd integer $m<a$. As the situation is symmetrical in $a$ and $b$, the theorem follows.

13.1. Proof of Theorem VI. It is enough to prove the theorem when $s=0$. In fact, $f-s$ and its Fourier series satisfy the conditions of the theorem for $s=0$. Thus, the truth of the theorem in this case implies that $f-s$ is continuous $(C ; a, b)$ with limit 0 . But this implies that $f$ is continuous $(C ; a, b)$ with limit $s$.

We shall consider the case of Cesàro summability. The proof for Riesz summability follows the same lines but requires one less step. We shall assume that $0<\alpha, 0<\beta$. The other cases can be treated in a similar fashion. The proof in all cases rests on the formula

$$
f_{a, b}(x, y)=\psi x^{a+\alpha+1} y^{b+\beta+1} \int_{T} H(x u) K(y v) \sigma_{\alpha, \beta}(u, v) d T .
$$

We reduce this formula in the case at hand to one involving Cesàro means by an application of (3.33). We have

$$
\sigma_{\alpha, \beta}(x, y)=\sum_{m<x} B(x-m) \sum_{n<y} C(y-n) S_{\alpha, \beta}(m, n),
$$

where

$$
\sum_{m<x}|B(x-m)| \leqq L, \quad \sum_{n<\nu}|C(y-n)| \leqq L,
$$

$L$ being a suitably chosen constant independent of $x, y$.

Let $0<\epsilon$ be given. We select a positive integer $m_{0}$ so that

$$
\left|S_{\imath, \beta}(m, n)\right| \leqq \epsilon m^{\alpha} n^{\beta}
$$

for $m_{0} \leqq m, m_{0} \leqq n$. We denote by $M$ a number independent of $x, y, m, n$, for $x \leqq \delta, y \leqq \delta, m_{0} \leqq m, m_{0} \leqq n$.

We now write 
1935]

DOUBLE FOURIER SERIES

433

$$
\begin{aligned}
\sigma_{\alpha, \beta}(u, v)= & \left\{-\sum_{m<m_{0}} \sum_{n<m_{0}}+\sum_{m<m_{0}} \sum_{n<v}+\sum_{m<u} \sum_{n<m_{0}}+\sum_{m_{0} \leqq m<u} \sum_{n_{0} \leqq n<v}\right\} \\
& \cdot B(u-m) C(v-n) S_{\alpha, \beta}(m, n) \\
= & Q_{1}+Q_{2}+Q_{3}+Q_{4},
\end{aligned}
$$

say, and

$$
\begin{aligned}
\int_{T} H(x u) K(y v) \sigma_{\alpha, \beta} d T= & \left\{\int_{(0,0)}^{\left(m_{0}, m_{0}\right)} \sigma_{\alpha, \beta}+\int_{\left(m_{0}, m_{0}\right)}^{(\infty, \infty)} Q_{1}\right\} H K d(u, v) \\
& +\left\{\int_{\left(0, m_{0}\right)}^{\left(m_{0}, \infty\right)} \sigma_{\alpha, \beta}+\int_{\left(m_{0}, m_{0}\right)}^{(\infty, \infty)} Q_{2}\right\} H K d(u, v) \\
& +\left\{\int_{\left(m_{0}, 0\right)}^{\left(\infty, m_{0}\right)} \sigma_{\alpha, \beta}+\int_{\left(m_{0}, m_{0}\right)}^{(\infty, \infty)} Q_{3}\right\} H K d(u, v) \\
& +\int_{\left(m_{0}, m_{0}\right)}^{(\infty, \infty)} H K Q_{4} d(u, v)=V_{1}+V_{2}+V_{3}+V_{4},
\end{aligned}
$$

say. Then

$$
\begin{aligned}
&\left|V_{1}\right| \leqq M+M \int_{m_{0}}^{\infty}|H(x u)| d u \int_{m_{0}}^{\infty}|K(y v)| d v \leqq M x^{-1} y^{-1}, \\
&\left|V_{2}\right| \leqq M \int_{m_{0}}^{\infty}|K| v^{\beta+1} d v+M \int_{m_{0}}^{\infty}|H| d u \int_{m_{0}}^{\infty}|K| v^{\beta+1} d v \leqq M x^{-1} y^{-\beta-2}, \\
&\left|V_{\mathbf{8}}\right| \leqq M x^{-\alpha-2} y^{-1}, \\
&\left|V_{4}\right| \leqq \epsilon L \int_{m_{0}}^{\infty}|H| u^{\alpha} d u \int_{m_{0}}^{\infty}|K| v^{\beta} d v \\
& \quad \leqq \epsilon L x^{-\alpha-1} y^{-\beta-1} \int_{0}^{\infty}|H(u)| u^{\alpha} d u \int_{0}^{\infty}|K(v)| v^{\beta} d v .
\end{aligned}
$$

In addition,

$$
\begin{aligned}
V_{2}= & \sum_{m<m_{0}} \int_{m}^{m_{0}} H(x u)(u-m)^{\alpha} d u \cdot \int_{m_{0}}^{\infty} K(y v) \sum_{n<v}(v-n)^{\beta} a_{m, n} d v \\
& +\sum_{m<m_{0}} \int_{m_{0}}^{\infty} H B(u-m) d u \cdot \int_{m_{0}}^{\infty} K \sum_{n<v} C(v-n) S_{\alpha, \beta}(m, n) d v \\
= & \sum_{p<2 m_{0}} g_{p}(x) G_{p}(y),
\end{aligned}
$$

say, where, for $p=0,1, \cdots, 2 m_{0}-1$,

$$
\left|g_{p}(x)\right| \leqq M x^{-1} \text {. }
$$


Consider $V_{2}$. We have

$$
\begin{aligned}
\left|x^{\alpha+1} y^{\beta+1} V_{2}\right| & \leqq M x^{-a} y^{-b}\left|f_{a, b}\right|+x^{\alpha+1} y^{\beta+1}\left(\left|V_{1}\right|+\left|V_{3}\right|+\left|V_{4}\right|\right) \\
& \leqq M\left(1+x^{\alpha} y^{\beta}+x^{-1} y^{\beta}+1\right) \leqq F(x),
\end{aligned}
$$

where $F$ depends upon $x$ but not upon $y$. Hence, since

$$
x^{\alpha+1} g_{p}(x)=O\left(x^{\alpha}\right)=o(1)
$$

as $x \rightarrow+0$, for $p=0,1, \cdots, 2 m_{0}-2$, we see, on replacing $x$ by $1 / x$ and $y$ by $1 / y$ in Lemma 4 , that

$$
V_{2}=o\left(x^{-\alpha-1} y^{-\beta-1}\right) \quad \text { as } \quad(x, y) \rightarrow(+0,+0) .
$$

In the same way we see that $V_{3}$ satisfies (13.11). On the other hand so also does $V_{1}$. Hence,

$$
\begin{aligned}
\limsup _{(x, y) \rightarrow(+0,+0)}\left|x^{-a} y^{-b} f_{a, b}(x, y)\right| & =\limsup _{(x, y) \rightarrow(+0,+0)}\left|x^{\alpha+1} y^{\beta+1} V_{4}\right| \\
& \leqq \epsilon L \int_{0}^{\infty}|H(u)| u^{\alpha} d u \int_{0}^{\infty}|K(v)| v^{\beta} d v .
\end{aligned}
$$

Since $\epsilon$ was arbitrary the theorem follows.

\section{BibliograPHY}

1. Agnew, R. P., On summability of double sequences, American Journal of Mathematics, vol. 54 (1932), pp. 648-656.

2. - On summability of multiple sequences, ibid., vol. 56 (1934), pp. 62-68.

3. Bosanquet, L. S., On the summability of Fourier series, Proceedings of the London Mathematical Society, vol. 31 (1930), pp. 144-164.

4. - Cesàro summation of Fourier series, ibid., vol. 35 (1934), pp. 17-32.

5. Hardy, G. H., and Littlewood, J. E., Solution of the Cesdro summability problem for power series and Fourier series, Mathematische Zeitschrift, vol. 19 (1923), pp. 67-96.

6. - The allied series of a Fourier series, Proceedings of the London Mathematical Society, vol. 24 (1926), pp. 211-246.

7. Hobson, E. W., Theory of Functions of a Real Variable, Cambridge, vol. I, 1927.

8. - Theory of Functions of a Real Variable, Cambridge, vol. II, 1926.

9. Kogbetliantz, Ervand, Sommation des séries et intégrales divergentes par les moyennes arithmétiques et typiques, Mémorial des Sciences Mathématiques, vol. 51 (1931), pp. 1-84.

10. Mears, Florence M., Riesz summabiliy for double series, Transactions of the American Mathematical Society, vol. 30 (1928), pp. 686-709.

11. Merriman, G. M., Concerning the summability of double series of a certain type, Annals of Mathematics, vol. 28 (1927), pp. 515-533.

12. - A set of necessary and sufficient conditions for Cesdro summability of double series, ibid., vol. 29 (1928), pp. 343-354.

13. Moore, C. N., On convergence factors in double series and double Fourier's series, Transactions of the American Mathematical Society, vol. 14 (1913), pp. 73-104.

14. Paley, R. E. A. C., On the Cesaro summability of Fourier series and allied series, Proceedings of the Cambridge Philosophical Society, vol. 26 (1930), pp. 173-203. 
15. Pollard, S., The summation of Denjoy-Fourier series, Proceedings of the London Mathematical Society, vol. 27 (1928), pp. 209-222.

16. Tonelli, L., Serie Trigonometriche, Bologna, 1928.

17. - Su un problema di Abel, Mathematische Annalen, vol. 99 (1928), pp. 183-199.

18. Wiener, N., A type of Tauberian theorem applying to Fourier series, Proceedings of the London Mathematical Society, vol. 30 (1929), pp. 1-8.

19. - Tauberian theorems, Annals of Mathematics, vol. 33 (1932), pp. 1-100.

20. Young, W. H., On multiple Fourier series, Proceedings of the London Mathematical Society, vol. 11 (1913), pp. 133-184.

21. - On infinite integrals involving a generalization of the sine and cosine functions, Quarterly Journal of Mathematics, vol. 43 (1912), pp. 161-177.

UNIVERSITY OF ROCHESTER, Rochester, N. Y.

United States Naval Academy, ANNAPOLIS, MD. 